Pietro Militello

\title{
Minoische Tradition und mykenische Innovation: Wandbilder und Kultaktivitäten in Agia Triada in SM IIIA*
}

\author{
Einführung
}

Das Kreta der Nachpalastzeit (SM II-III) hat besonders in den vergangenen Jahren in der Forschung, die stets auf das neopalatiale Kreta fokussiert war, verstärkt Aufmerksamkeit erfahren ${ }^{1}$. Dies ist wahrscheinlich als Folge zweier gleichzeitiger Phänomene zu erklären: erstens wegen des allgemeinen wachsenden Interesses an der Geschichte des Mittelmeergebietes in der zweiten Hälfte des 2. und zu Beginn des 1. Jahrtausends v. Chr. und zweitens aufgrund der Aufmerksamkeit für die Phänomene kultureller Interaktion. Es besteht kein Zweifel daran, dass das größte Forschungsproblem des Zeitraumes SM II-III nicht nur die politischen, sondern auch die kulturellen und gesellschaftlichen Beziehungen zwischen Kreta und dem Festland, d. h. zwischen minoischer und mykenischer Tradition darstellen. Nach der Zerstörung der minoischen Paläste am Ende von SM I, um 1430 v. Chr., stand die Insel unter dem politischen Einfluss des Festlandes, sodass wir für diesen Zeitpunkt vom 'mykenischen' Kreta sprechen können². Wie aber dieses 'mykenische' Kreta wirklich zu verstehen ist, und wie die Gesellschaft dieser Zeit strukturiert war, ist nicht leicht nachzuvollziehen. Die einzig belegbare Tatsache ist die Existenz einer mykenischen Verwaltung in Knossos, welche durch die Linear-BTafeln bezeugt wird. Wie lange aber diese Verwaltung bestand, ist noch umstritten, zumal die Chronologie

\footnotetext{
* Dieser Artikel ist die erweiterte Fassung eines Vortrags, den ich auf dem von F. Blakolmer organisierten 2. Ägäischen Kolloquium am 20. Oktober 2005 in Wien gehalten habe. Ich möchte F. Blakolmer für die Einladung danken, an dieser Tagung teilzunehmen, und F. Krinzinger für die Möglichkeit, den Beitrag in der vorliegenden Zeitschrift zu publizieren. Die Übersetzung meines Textes verdanke ich Frau N. Hartung. - Außer den in den ÖJh üblichen Sigeln werden hier die folgenden Abkürzungen verwendet: Burke 2005 = B. Burke, Materialization of Mycenaean Ideology and the Ayia Triada Sarcophagus, AJA 109, $2005,403-422$. Cucuzza 2003 = N. Cucuzza, Il volo del grifo: osservazioni sulla Haghia Triada 'micenea', Creta Antica 4, 2003, 199-272. La Rosa 1999 = V. La Rosa, Nuovi dati sulla tomba del Sarcofago Dipinto di Haghia Triada, in: V. La Rosa - D. Palermo L. Vagnetti (Hrsg.), Epi ponton plazomenoi. Simposio italiano di studi egei dedicato a L. Bernabò Brea e G. Pugliese Carratelli, Roma 1998 (1999) 177-188.

Militello 1998 = P. Militello, Haghia Triada I. Gli Affreschi, Monografie della Scuola archeologica di Atene e delle missioni italiane in Oriente 9 (1998).

Paribeni 1908 = R. Paribeni, Il sarcofago dipinto di Haghia Triada, MonAnt 19, 1908, 5-87.

PM I-IV = A. Evans, The Palace of Minos at Knossos I-IV (1921-1935).

${ }^{1}$ s. z. B. verschiedenen Kongresse, die im vergangenen Jahrzehnt dem SH-III-zeitlichen Kreta gewidmet waren: J. Driessen - A. Farnoux (Hrsg.), La Crète mycénienne. Table Ronde, Athènes 1991, 30. Suppl. BCH (1997); B. P. Hallager - E. Hallager (Hrsg.), Late Minoan III Pottery: Chronology and Terminology. Acts of the Meeting Athens 1994, Monographs of the Danish Institute at Athens 1 (1997); A. L. D’Agata - J. Moody (Hrsg.), Ariadne’s Threads. Connections between Crete and the Greek Mainland in Late Minoan III (LM IIIA2 to LM IIIC). Proceedings of the Workshop Athens 2003, Tripodes 3 (2005). Im Bereich der Monographien s. z. B. W. Löwe, Spätbronzezeitliche Bestattungen auf Kreta, BAR International Series 642 (1996) und E. Borgna, Il complesso di ceramiche TM III dall'Acropoli Mediana di Festòs, Studi di Archeologia Cretese III (2003).

2 s. z. B. Driessen - Farnoux (Anm. 1). Weitere Bemerkungen über den Gebrauch und die Grenzen dieser Definition in D’Agata Moody (Anm. 1) 9-16.
} 

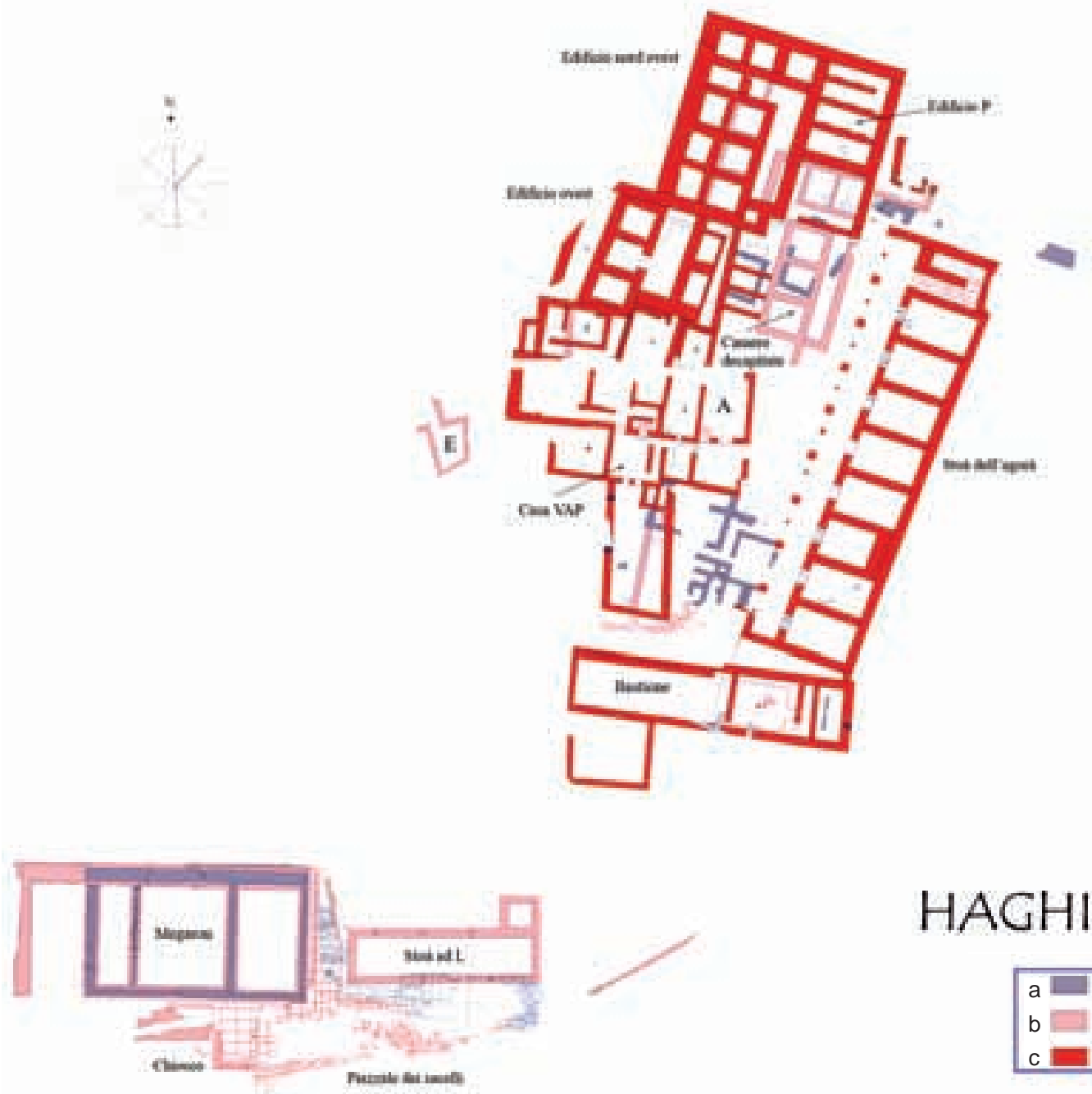

\section{HAGHIA TRIADA}
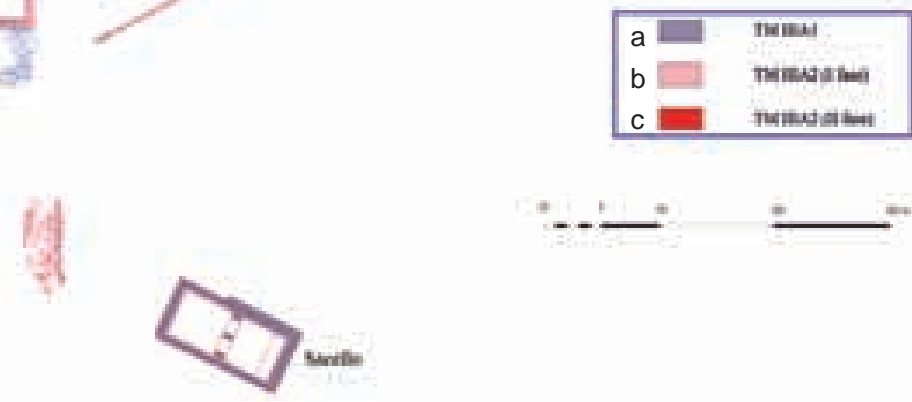

1a-c Agia Triada in SM III (a: erste Phase: SM IIIA1; b: zweite Phase: SM IIIA2; c: dritte Phase: SM IIIA2) 
der knossischen Stratigraphien unsicher ist ${ }^{3}$, und unklar sind auch das Ausmaß der mykenischen Präsenz und die Tiefe ihrer Verwurzelung in der Gesellschaft Kretas ${ }^{4}$.

Aus dieser Sichtweise möchte ich mich in diesem Beitrag behutsam dem Problem des Verhältnisses zwischen Tradition und Innovation in SM III annähern. Ich werde mich nur mit einer einzelnen Siedlung, Agia Triada, in dem kurzen Zeitabschnitt SM IIIA1-2 Früh befassen, und dies unter einem besonderen Aspekt, nämlich jenem der Religion und der Malerei. Religion als Rechtfertigung der Macht und Wandmalerei als Medium ideologischer und politischer Propaganda sind zwei der wichtigsten Bereiche, in welchen interkulturelle Beziehungen, besonders zwischen einer politisch dominanten und einer unterworfenen Kultur, reflektiert werden. Insbesondere die Freskomalereien im SM-III-zeitlichen Agia Triada sind in Hinblick sowohl auf die Bildthemen wie auch auf die Funktionen als Ausdruck der öffentlichen Religion zu verstehen. Nach einer kurzen Beschreibung der SM-III-Siedlung, der Malereien und der Fundobjekte religiösen Charakters wird hier das Verhältnis zwischen den auf Fresken dargestellten und tatsächlich praktizierten Ritualen untersucht, um die Neuerungen in den kultischen Handlungen herauszuarbeiten und letztendlich deren Bedeutung zu verstehen.

\section{Die Siedlung von Agia Triada}

Am Ende der Periode SM IB, nach der neupalastzeitlichen Blüte, als Agia Triada Sitz einer Villa und eines bedeutenden zeremoniellen und wirtschaftlichen Zentrums war, wurde die Siedlung durch einen Brand zerstört ${ }^{5}$. Dabei handelte es sich aber nicht um eine einzige Zerstörungsschicht, sondern um die Folge von mindestens zwei oder drei Ereignissen, die zuerst, gegen Ende von SM IB, die Villa, danach die 'Casa Est' und schließlich die 'Bastione’ betrafen ${ }^{6}$. Der Wiederaufbau der SM-III-Siedlung erstreckte sich bis SM IIIA2 und kann in drei Phasen unterteilt werden'.

In der ersten Phase (Abb. 1a) während SM IIIA1 wurden im Süden das große Megaron und der Schrein, das sog. Sacello, im Norden die 'Casa con la gourna', die 'Casa delle Sfere Fittili' und kleinere Räume erbaut, während im Süden die 'Bastione’ und im Nordosten die alte Tholos B mit zwei Gräbern wiederverwendet wurden. In der zweiten Phase (Abb. 1b), am Beginn von SM IIIA2, wurden im Süden die kleine 'Stoa FG' und der Kiosk - eine Art Pavillon mit Säulen und einer Bank, der an das Megaron angebaut wurde, - und im Norden das 'Haus der enthaupteten Zimmer' (Casa delle Camere decapitate) errichtet. Im Areal der älteren 'Casa del Lebete’ wurde der Raum E als sakraler Ort genutzt. In dieselbe Periode sind zudem das ‘Grab des

\footnotetext{
${ }^{3}$ Nach der bahnbrechenden Untersuchung von J. Driessen ist die 'Einheit der Archive' verfälscht worden und die verschiedenen Depots von Linear-B-Täfelchen sind in verschiedenen Zerstörungshorizonten anzunehmen: J. Driessen, An Early Destruction in the Mycenaean Palace at Knossos (1990); ders., Le palais de Cnossos au MR II-III. Combiens des destructions?, in: Driessen - Farnoux (Anm. 1) 113-134; R. Firth, A review of the Find-Places of the Linear B Tablets from the Palace of Knossos, Minos 35/36, 2000/01, 63-290. Während eine Gruppe der Täfelchen ('Room of the Chariot Tablets') in SM II-IIIA1 gesetzt werden kann, trifft eine Datierung des Großteils der anderen Gruppen in SM IIIA2 oder SM IIIB früh zu. Im Allgemeinen werden jedoch eine große Zerstörung und ein starker Wandel der politischen Situation Kretas in SM IIIA2 früh von allen Forschern akzeptiert; s. z. B. die Themenwahl der Herausgeber des Kolloquiums »Ariadne’s Threads« (Anm. 1), die SM IIIA2 als terminus post quem wählten.

${ }^{4}$ s. die von L. Baumbach, J. Driessen und besonders P. Ilievsky fortgeführte Analyse der in den Linear-B-Täfelchen genannten Anthroponyma, die eine »integrated society « bezeugen: L. Baumbach, An Examination of the personal Names in the Knossos Tablets as Evidence for the social Structure of Crete in LM II Period, in: O. Krzyszkowska - L. Nixon (Hrsg.), Minoan Society. Proceedings Cambridge Colloquium 1981 (1983) 3-10; dies., The People of Knossos: further Thought on some of the personal Names, in: J.-P. Olivier (Hrsg.), Mykenaikà. Actes X Colloque Mycénien, Athens 1990, 25. Suppl. BCH (1992) 57-64; P. H. Ilievsky, Observations on the personal Names from the Knossos D Tablets, in: Olivier a. O. 321-350; J. Driessen, The Scribes of the Room of the Chariot Tablets (2000) 189-194.

${ }^{5}$ Zusammenfassung zur Siedlungsgeschichte: V. La Rosa, Ayia Triada, in: J. Wilson - E. E. Myers - G. Cadogan (Hrsg.), The Aerial Atlas of Ancient Crete (1992) 70-77, und bes. V. La Rosa (Hrsg.), Per il centenario dello scavo di Haghia Triada. 1902-2002, Creta Antica 4 (2003).

${ }^{6}$ D. Puglisi, Haghia Triada nel periodo Tardo Minoico I, Creta Antica 4, 2003, 145-198 bes. 188-191.

7 Zur Siedlung in SM III s. V. La Rosa, Haghia Triada à l'époque mycénienne: L'utopie d'une ville capitale, in: Driessen - Farnoux (Anm. 1) 249-266; Cucuzza 2003. Cucuzza 2003, 220 unterscheidet zwei architektonische Hauptphasen, deren erste in zwei Subperioden gegliedert werden kann.
} 
bemalten Sarkophags' und eventuell ein drittes Grabmal in Form einer Grabgrube, die zwischen diesem und der Tholos B freigelegt wurde, zu datieren ${ }^{8}$. Später in SM IIIA2 wurde im nördlichen Sektor eine vollständige Renovierung vorgenommen, welche die dritte Phase bildet (Abb. 1c). Um die Errichtung der 'Großen Stoa' zu ermöglichen, wurden die Hänge des Hügels und folglich auch das 'Haus der enthaupteten Zimmer' eingeebnet. Die öffentlichen Funktionen des zerstörten Gebäudes wurden wahrscheinlich auf zwei neue Bauten übertragen, die denselben regelmäßigen Grundriss aufweisen: das 'Edificio Ovest' ('Westbau') und das 'Edificio NW/P' ('Nordwestbau'). Ein neues Haus, die 'Casa VAP' ('Casa dei vani aggiunti progressivamente'), wurde errichtet. Gerade in dieser Zeit aber wurde das 'Grab des bemalten Sarkophags' gestört und seine Beigaben wurden in die sog. Tomba degli Ori geworfen. Zugleich fiel der Schrein einem Brand zum Opfer'. Nach dessen Renovierung präsentierte sich die Siedlung wie eine kleine Zitadelle. Der größte Teil des Ortes bestand aus öffentlichen Gebäuden, die nach einem Plan angelegt worden waren, der spezielle Abschnitte vorsah: sakrale Bauten im Südosten, Herrschaftsgebäude im Südwesten und Verwaltungsgebäude im Norden. Es gab nur wenige Privathäuser, vermutlich nur ein einziges großes. Agia Triada erscheint nun wie eine »hauslose Stadt«, wie kürzlich formuliert wurde ${ }^{10}$. Am Ende von SM IIIA2 wurde auch die Art und Weise dieser strengen Stadtplanung aufgehoben. In der zweiten Hälfte des 13. Jahrhunderts wurde die Siedlung endgültig verlassen und nur der südliche Teil, die sog. Piazzale dei Sacelli, weiterhin als Heiligtum für kultische Aktivitäten im Freien genutzt ${ }^{11}$.

\section{Die Fresken aus SM III}

Vier Freskengruppen gehören der ersten und zweiten Periode der Nachpalastzeit an: der bemalte Fußboden des Schreins, die 'Kleine Prozession', der prominente bemalte Sarkophag und die Fragmente aus dem sog. Freskenschutt ${ }^{12}$.

Tabelle 1: Chronologie der Fresken von Agia Triada

\begin{tabular}{|l|l|}
\hline SM II/IIIA1 & 'Kleine Prozession’ \\
\hline SM IIIA1 - I. Bauphase & Fragmente aus dem ‘Freskenschutt’, bemalter Fußboden \\
\hline SM IIIA2 - II. Bauphase & Steinsarkophag \\
\hline SM IIIA2 - III. Bauphase & Entfernung der Malereien \\
\hline
\end{tabular}

${ }^{8}$ In diese Periode werden jetzt auch die Räume X, Y, und Z datiert. V. La Rosa - S. Privitera, La fascia subito a Nord del cd. Muraglione a Denti ad Haghia Triada, ASAtene 81, 2003, 735-768.

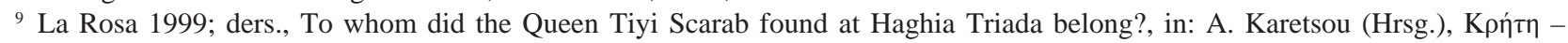

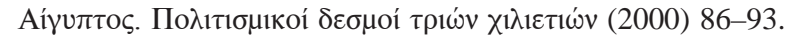

${ }^{10}$ Die Definition »houseless town« stammt von S. Privitera, Looking for a home in a houseless town, in: K. Glowacki - N. VogeikoffBrogan (Hrsg.), The Archaeology of House and Household in Crete, Congress Hierapetra 2005 (in Druck).

${ }^{11}$ Die Struktur der Piazzale und die Hunderten dort gefundenen Tonidole wurden von A. L. D’Agata rekonstruiert und publiziert: A. L. D’Agata, Haghia Triada II. Statuine minoiche e postminoiche dai vecchi scavi di Haghia Triada (1999); s. auch dies., Religion, Society and Ethnicity on Crete at the End of the Late Bronze Age. The Contextual Framework of LM IIIC Cult Activities, in: R. Laffineur - R. Hägg (Hrsg.), Potnia: Deities and Religion in the Aegean Bronze Age. Proceedings of the 8th International Aegean Conference Göteborg 2000, Aegaeum 22 (2001) 345-354. Weitere Beobachtungen dazu: D. Palermo, Haghia Triada tra il XII ed il VII secolo a.C., Creta Antica 4, 2003, 273-285. Zum griechischen und hellenistischen Agia Triada: V. La Rosa, Considerazioni sul sito di Haghia Triada in età ellenistico-romana, ASAtene 66/67, 1988/89 (1993) 259-275; V. La Rosa, Il cimitero veneziano di Haghia Triada, Creta Antica 2, 2001, 237-290.

12 Nach Knossos stellen diese Fresken die zweitgrößte Fundmenge von »mykenischen Malereien« aus Kreta dar. Trotzdem wurden nur wenige Abbildungen von Paribeni publiziert (Paribeni 1908, 67-68 Abb. 21-23) und fanden somit nur beschränkt Eingang in die Literatur. Der Mangel an Interesse für diese Funde erklärt sich wahrscheinlich durch deren grobe Herstellungsweise, besonders im Vergleich zu jenen des vorhergehenden Zeitabschnitts, und durch das allgemeine Desinteresse der Forschung für die Spätbronzezeit III. Die Fresken wurden publiziert in: Militello 1998. Dieser Monographie sind auch die näheren Daten zu Chronologie und Fundorten zu entnehmen. 
Tabelle 2: Chronologie der Funde

\begin{tabular}{|c|c|}
\hline I. und II. Phase & III. Phase \\
\hline Schrein (Phase I) & Schrein (Phase II-III) \\
\hline Megaron & Megaron \\
\hline 'Vano E' & 'Casa VAP', 'Vano A' \\
\hline & Agorà Est \\
\hline Grab des Sarkophags (?) & Areal der 'Tomba degli Ori' (Wiederbenutzung) \\
\hline
\end{tabular}

\section{Der bemalte Fußboden}

Das einzige Beispiel, dessen ursprüngliche Position mit Sicherheit bestimmt werden kann, ist der bemalte Fußboden des Schreins $\mathrm{H}^{13}$ (Abb. 2). Das Gebäude wurde in SM IIIA1 errichtet und bildete einen rechteckigen, von Nordwesten nach Südosten orientierten Bau, dessen Längsachse exakt mit der Position des Sonnenuntergangs im Sommer übereinstimmte. Es bestand aus zwei Räumen, einem Vorraum und einem inneren, mit einer Bank versehenen Raum, die durch ein Polythyron miteinander verbunden waren. Im inneren Raum ließen sich drei verschiedene Phasen unterscheiden ${ }^{14}$ : In der ersten wurden der Fußboden und der Sockel mit figürlichen Motiven dekoriert. Nach der Zerstörung durch einen Brand wurde der Boden um 7-15 cm erhöht und einfach verputzt. In der Füllschicht wurden drei 'Snake Tubes', drei konische Becher und drei Kalathoi gefunden. Reste von Glaspaste mit Wellendekor und ein goldenes Plättchen gehörten wohl zu einer hölzernen Pyxis. Der neue Fußboden bestand aus einfachem Stampflehm, während die Wände und die Bank einen schlichten weißen Verputz mit einem roten Streifen aufwiesen; auf dem neuen Fußboden wur-

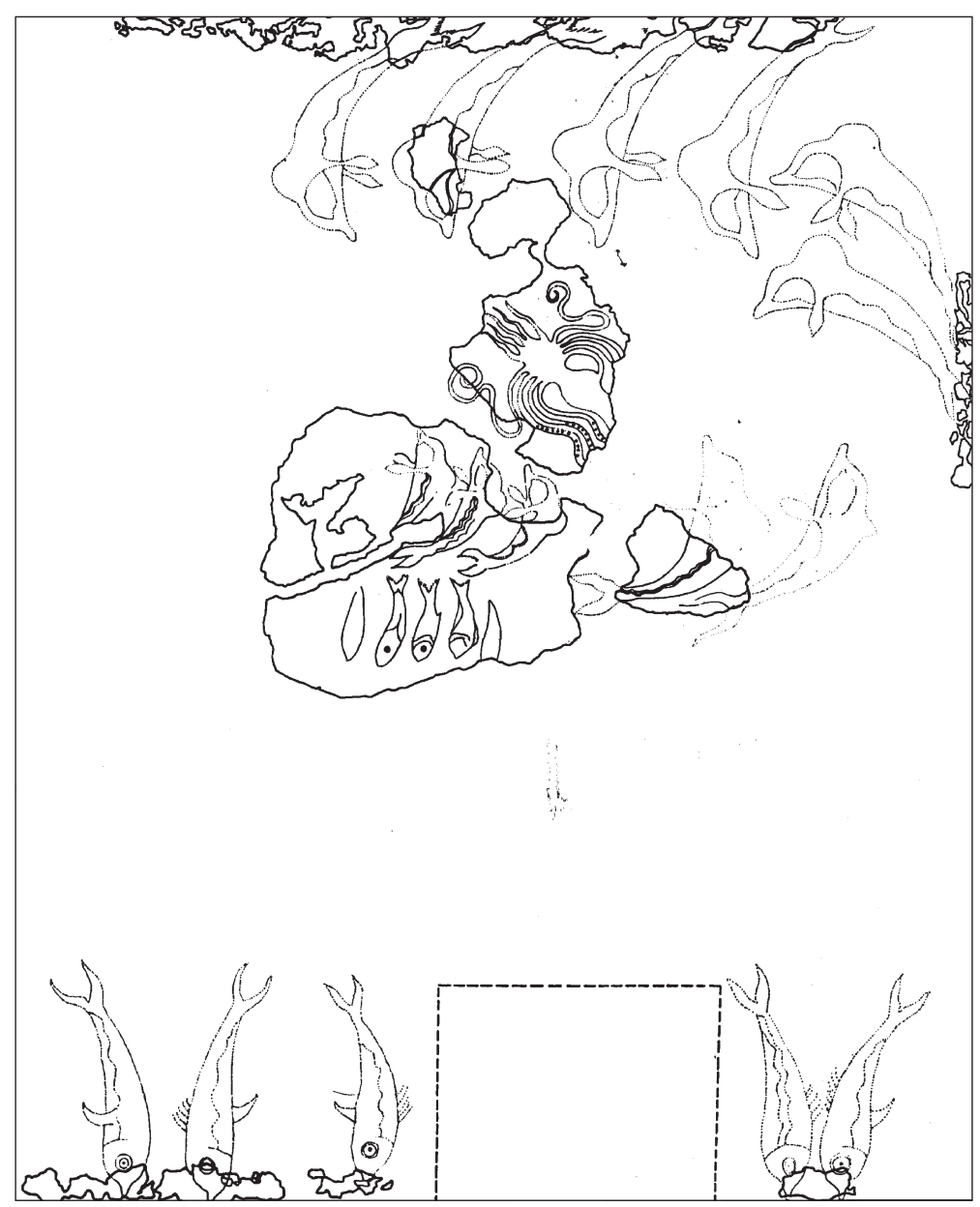

2 Schrein H von Agia Triada, Planimetrie mit dem bemalten Fußboden den die Fragmente eines 'Snake Tube' entdeckt. In einer dritten Phase wurde der Fußboden erneut aufgeschüttet. Auch aus dieser Schicht stammen drei 'Snake Tubes' mit schlangenförmigen Appliken und eine Lampe, die auf dem Fußboden gefunden wurden, sowie eine große Menge von Schalen und Miniaturamphoren, welche umgedreht, d. h. auf dem Kopf stehend, auf der Bank angetroffen wurden.

In der ersten und prächtigsten Phase war der Fußboden mit einer Meeresszene bemalt, während der Sockel aus imitierten Marmorplatten bestand. Das Fresko dehnte sich nahezu über den gesamten Boden aus. Eine

\footnotetext{
${ }^{13}$ Militello 1998, 148-153. 321-335 Die SM-III-Chronologie des Gebäudes wurde durch die neuen Ausgrabungen bestätigt. Ursprünglich war es in SM I datiert worden.

${ }^{14}$ L. Banti, I culti minoici e greci di H. Triada (Creta), ASAtene n. s. 3/4, 1941/43, 9-74.
} 
von der Bemalung ausgesparte, rechteckige Fläche an der Südseite des Raumes lässt hier auf einen rechteckigen Gegenstand schließen, der auch eine entsprechende Fläche an der Wand vor dem Brand schützte. Aufgrund der Form und der Position können wir dieses Objekt als Thron definieren, der den festländischen Exemplaren ähnelte ${ }^{15}$.

Die nur fragmentarisch erhaltene Bemalung des Fußbodens besaß zwei Kompositionszentren. Im Norden befanden sich ein Oktopus und mehrere Delphine, die eine radiale Komposition mit dem Oktopus im Zentrum bildeten, im Süden war eine Reihe großer Fische, vermutlich Thunfische, entlang der Bank angeordnet. Leider fehlen große Teile, sodass unbekannt bleibt, was im Zentrum dargestellt war; die kleineren Fische scheinen eine Verbindung zwischen den zwei Szenen herzustellen. Bemerkenswert ist, dass die Hauptmotive nicht in der Raummitte, sondern links und rechts der Mittelachse in der Verlängerung des Einganges zu finden sind. Somit liegt die Annahme nahe, dass diese Komposition nicht für einen im Inneren des Raumes befindlichen Betrachter gedacht war, sondern für denjenigen, der den Raum betrat.

Die im Fußbodenfresko dargestellten Oktopoden und Delphine haben eine lange Tradition in der minoischen Ikonographie ${ }^{16}$, wobei der Delphin jedoch außerhalb Kretas eine größere Beliebtheit genossen zu haben scheint: Wir finden ihn etwa in den Fresken von Thera und als Emblem der peloponnesischen Dolche in Niellotechnik ${ }^{17}$. Die Kombination beider Tiere ist aber eine Neuerung, die um 1400 v. Chr. auf der Goldschale von Dendra eingeführt wurde ${ }^{18}$ und nur symbolische Bedeutung haben konnte, zumal beide Wesen in unterschiedlichen ökologischen Systemen leben und somit nie gemeinsam anzutreffen sind. Diese motivische Zusammenführung, über deren Bedeutung wir nur spekulieren können, ist ein Merkmal der mykenischen Hochkultur und erreichte in den bemalten Fußböden der mykenischen Paläste ihren Höhepunkt. Es ist überlegenswert, ob die Verbindung dieser beiden Tiermotive nicht vielleicht den Zusammenschluss von politischem und religiösem Bereich oder jenen der minoischen und der mykenischen Tradition symbolisierte.

\section{Die 'Kleine Prozession'}

Die 'Kleine Prozession' (Abb. 3) ist ein überaus interessantes Wandbild, dessen genaue Fundstelle leider unbekannt bleibt ${ }^{19}$, weshalb seine Datierung in die Nachpalastzeit allein auf stilistischen Merkmalen beruht. Es handelt sich um ein von Balkenkopfreihen gerahmtes und von einem dreiteiligen Reliefbalken mit 'Kulthörnern' bekröntes Bild mit einer Ausdehnung von ca. $62 \times 72 \mathrm{~cm}$. Das innere Feld wird von zwei weißen und roten Streifen in zwei übereinanderliegende Figurenfriese geteilt. Auf dunkelgelbem Hintergrund stellt der obere Fries fünf nach links schreitende Frauen dar $^{20}$, deren linken Arme vorgestreckt sind, während ihre

\footnotetext{
15 Militello 1998, 329; Cucuzza 2003, 260 f.

${ }^{16}$ s. die Diskussion bei Militello 1998, 321-327; s. auch ders., L’ambiguo regno di Minosse, in: A. Giannattasio (Hrsg.), Mare, jam, pontos, Convegno Genova 2004 (2006) 155-178.

17 Vgl. Wandmalereien im theräischen 'Westhaus': C. Doumas, The Wall-Paintings of Thera (1992) Taf. 35-36; Dolche: A. XenakiSakellariou - C. Chatziliou, 'Peinture en métal’ à l'époque mycénienne (1989) 27 Kat. 8 (Prosymna); 28 Kat. 11 (KatarraktisPharai).

18 S. Marinatos - M. Hirmer, Kreta, Thera und das Mykenische Hellas (1973) Taf. 218-219.

19 Militello 1998, 142-148. 309-320.

${ }^{20}$ Die Identifikation der Figuren als Frauen hängt von der Konvention der weißen Farbe für das Inkarnat ab. In der ägäischen Ikonographie ist diese von ägyptischen Vorbildern abgeleitete Regel seit Langem anerkannt worden; s. z. B. S. Hood, The primitive Aspects of Minoan artistic Conventions, in: P. Darcque - J.C. Poursat (Hrsg.), L’iconographie minoenne. Actes de la Table Ronde d'Athènes 21-22 avril 1983, 11. Suppl. BCH (1985) 21-27. In den letzten Jahren sind aber einige Argumente gegen die allgemeine Gültigkeit dieser Konvention erhoben worden: F. Blakolmer, Überlegungen zur Inkarnatsfarbe in der frühägäischen Malerei, ÖJh 62, 1993, 5-18; B. Olsen, Women, Children and the Family in the Late Bronze Age: Differences in Minoan and Mycenaean Constructions of Gender, World Archaeology 29, 1998, 380-392; S. Langdon, Figurines and Social Change: Visualizing Gender in Dark Age Greece, in: N. Wicker - B. Arnold (Hrsg.), From the Ground Up: Beyond Gender Theory in Archaeology. Proceedings of the Fifth Gender and Archaeology Conference, University of Wisconsin-Milwaukee, 1998 (1999) 23-29; Ch. Doumas, Age and Gender in the Theran Wall Paintings, in: S. Sherratt (Hrsg.), The Wall Paintings of Thera. Proceedings of the First International Symposium, Thera 1997, II (2000) 971-981; B. Alberti, Gender and the Figurative Art of Late Bronze Age Knossos, in: Y. Hamilakis (Hrsg.), Labyrinth Revisited. Rethinking 'Minoan’ Archaeology (2002) 98-117. Es ist aber immer möglich, Gründe zu finden, die Ausnahmen rechtfertigen; seien es Knaben oder Mädchen, die während Transitionsriten ihre endgültige sexuelle Identität in der Gesellschaft noch nicht ausgeprägt haben, sei es, dass die Wiedergabe eines Dekormotivs und
} 
rechten Hände - in der konventionellen Haltung von Betenden - an die Stirn geführt werden. Zwischen zwei Frauen steht jeweils ein kleiner Pfeiler mit Voluten- und Papyruskapitell in Form von A. Evans' 'waz'Motiv. Der untere Fries besitzt heute dunkelvioletten Grund, der ursprünglich blau gewesen sein dürfte. Sieben oder acht weibliche Figuren schreiten von rechts nach links, und in diesem Fries hat jede von ihnen ihren rechten Arm auf die Schulter der Vorangehenden gelegt, während der linke Arm an der Hüfte ruht. Die Frisuren der Frauen beider Gruppen sind unterschiedlich: Die Figuren des oberen Frieses tragen eine dunkelviolette

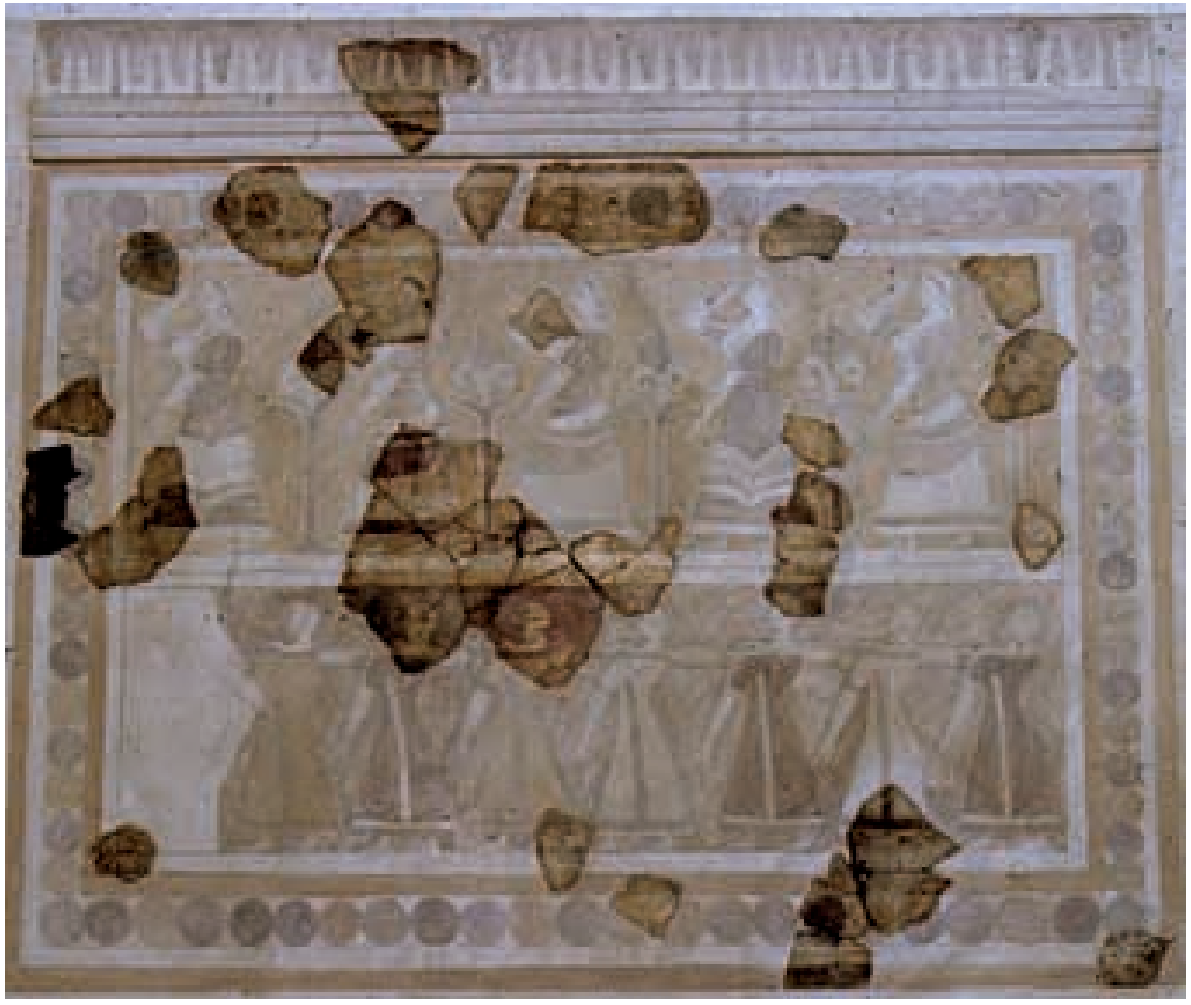

3 ‘Kleine Prozession’ aus Agia Triada Binde um den Kopf, die von der Stirn auf die Schulter fällt, wobei eine weiße Stelle an der Spitze des Kopfes ausgespart ist. Die Köpfe der Frauen im unteren Fries dagegen sind vollständig rasiert. Alle Figuren tragen den typischen langen minoischen Rock und ein vorn offenes, die Brüste unbedeckt lassendes Oberteil. Die Brüste der Frauen im oberen Fries sind üppiger, während jene der Frauen im unteren Paneel nicht zu sehen sind ${ }^{21}$.

In diesem Wandgemälde begegnen mehrere eigenartige Elemente, so die parataktische Anordnung der Figuren und die Kompositionsweise übereinander liegender Friese ${ }^{22}$. Bemerkenswert ist ferner die dargestellte Lokalität. Wir können nicht ausschließen, dass der Rahmen des Bildes nur eine dekorative Funktion besaß, und in diesem Fall wären die 'Kulthörner' bloß ein Kennzeichen des religiösen Charakters der abgebildeten Szene. Man könnte hingegen aber auch vermuten, dass die Balkenköpfe ihrer ursprünglichen Bedeutung folgend ein Architekturbild wiedergeben und die dargestellten Handlungen demnach in einem zweigeschossigen Gebäude geschildert werden. Folgerichtig hätten wir die 'Kulthörner' als Dachbekrönung dieses Gebäudes zu verstehen, wie dies auch in zahlreichen Abbildungen minoischer Palastarchitektur bezeugt ist.

nicht einer Person vorliegt, wie z. B. in der Textilkunst. Gerade diese Ausnahmen bezeugen deshalb nicht das Fehlen einer Regel, sondern die Komplexität des semantischen Gebrauchs der Farben.

${ }^{21}$ Dies kann zwar nicht mit Sicherheit nachgewiesen werden, die Figuren sind jedoch mit stark gebeugten Schultern dargestellt, sodass man nicht ausschließen kann, dass die Brüste hinter der Schulter versteckt sind. Es ist aber zu anzumerken, dass dieselbe Art der Darstellung bei den Frauenkörpern des oberen Frieses angewandt wurde, wobei die gesamte Brust sichtbar wird.

${ }^{22}$ Militello 1998, 154-167. 


\section{Der bemalte Sarkophag}

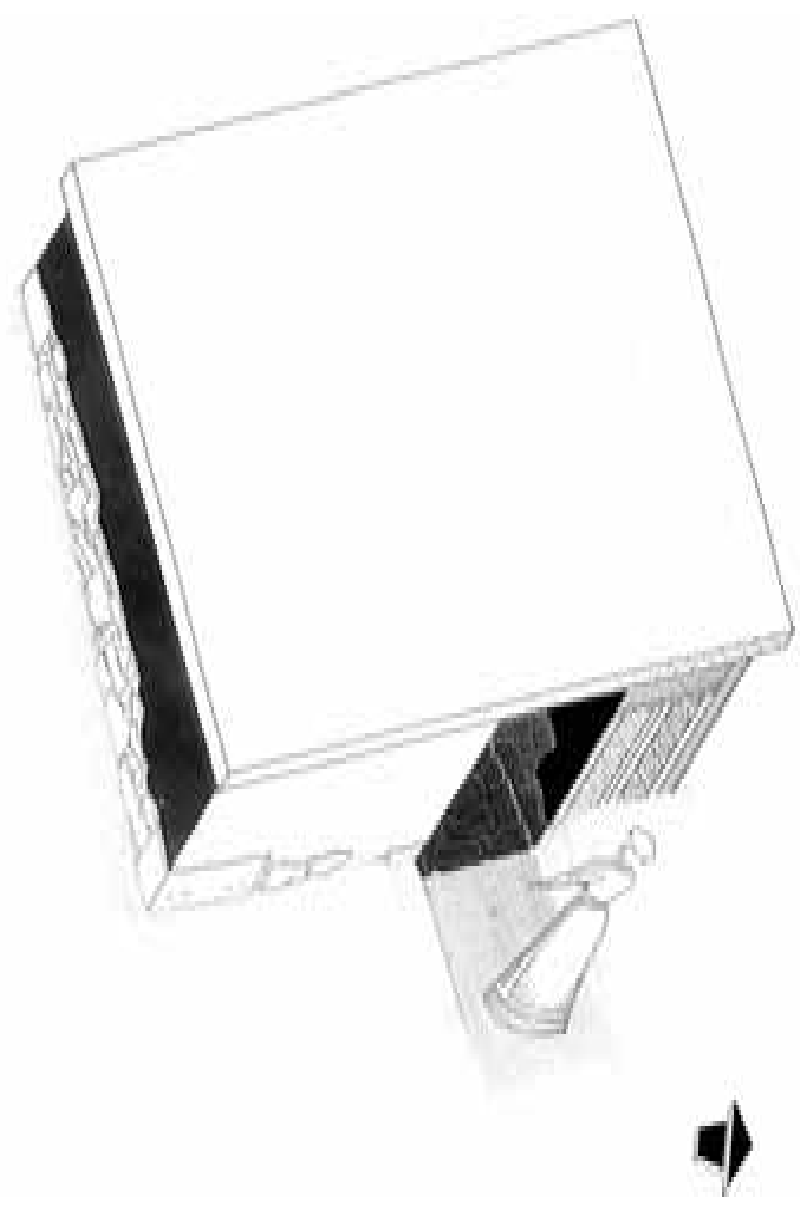

4 Grab des bemalten Sarkophags in Agia Triada, Rekonstruktion
Der berühmte Steinsarkophag von Agia Triada mit seinem Freskenschmuck ${ }^{23}$ wurde in einem Grab gefunden, das wie ein rechteckiger, teilweise unterirdischer Bau aussah und seinen Eingang an einer Schmalseite unter Bodenniveau hatte (Abb. 4) ${ }^{24}$. Die Auffindungssituation ist aufgrund der veröffentlichten Beschreibung gut bekannt ${ }^{25}$ : Der Sarkophag lag zur Seite gekippt. In einer Grube wurde ein zweiter tönerner Sarkophag gefunden, der chronologisch jedoch später anzusetzen ist. Man gewinnt den Eindruck, das Grab wäre beraubt und nur wenige Objekte wären zurückgelassen worden. Abgesehen von den Beigaben des Tonsarkophags wurden ein Rasiermesser, ein Steingefäß und eine Tritonmuschel entdeckt; darüber hinaus können dank der Tagebuchaufzeichnungen der Ausgräber ein Tonidol und eine rote Schale diesem Grab zugeschrieben werden ${ }^{26}$. Aus ihm könnten auch weitere Beigaben stammen, die in der nahe gelegenen 'Tomba degli Ori' gefunden wurden: Unter ihnen befanden sich der berühmte Skarabäus der ägyptischen Königin Teje und eine hethitische Sphingenfigur ${ }^{27}$. Die Fragmente zweier Tassen im Verband der Steinmauer geben uns die genaue chronologische Einordnung der Bauzeit an: Sie stammen aus demselben Zerstörungshorizont, welcher in Knossos die Übergangszeit von SM IIIA1 zu SM IIIA2 bildet ${ }^{28}$. Dieser ungewöhnliche Grabtypus in Agia Triada entspricht dem nicht minder ungewöhnlichen Bestattungstypus des Sarkophags, und folglich können wir dieselbe Zeitstellung auch für den Sarkophag annehmen.

Die Steinoberflächen des Sarkophags wurden mit einer dünnen Schicht überzogen und danach in Freskotechnik bemalt. Dieses Bildwerk ist überaus bekannt, soll hier aber dennoch kurz beschrieben werden.

${ }^{23}$ Die Bibliographie zum bemalten Sarkophag von Agia Triada ist in der Zwischenzeit unüberschaubar geworden. Nach der grundlegenden Diskussion von Ch. Long, The Ayia Triada Sarkophagus (1974) (mit älterer Bibliographie) wurde der Sarkophag noch mehrmals analysiert: R. Laffineur, A propos du sarcophage d'Aghia Triada: un rituel de nécromancie à l'époque protohistorique?, Kernos 4, 1991, 277-285; W. Löwe, Spätbronzezeitliche Bestattungen auf Kreta, BAR International Series 642 (1996) 23-41; N. Marinatos, Minoan sacrificial Ritual. Cult Practice and Symbolism (1986) 25-27; W. Pötscher, Der Termin des Festes auf dem Sarkophag von Hagia Triada, Klio 76, 1994, 67-77; ders., Zum Sarkophag von Hagia Triada, in: E. De Miro - L. Godart - A. Sacconi (Hrsg.), Atti e memorie del secondo Congresso internazionale di micenologia, Roma-Napoli 1991, II (1996) 857-859; ders., Tag und Nacht auf dem Sarkophag von Hagia Triada, Klio 79, 1997, 19-22 und 315 f.; ders., Bemerkungen zum Sarkophag von Hagia Triada, in: F. Blakolmer (Hrsg.), Österreichische Forschungen zur Ägäischen Bronzezeit 1998. Akten der Tagung Wien 1998, WForsch 3 (1998) 107 f.; St. Hiller, Egyptian Elements on the Hagia Triada Sarcophagus, in: P. Betancourt u. a. (Hrsg.), Meletemata. Studies in Aegean Archaeology Presented to Malcolm H. Wiener II, Aegaeum 23 (1999) 361-369; Burke 2005.

${ }^{24}$ Paribeni 1908. Die Zeichnung von Paribeni a. O. 7 Abb. 1 stellt die Mauerstruktur in einer regelmäßigen Technik dar. Wahrscheinlich wurde sie nicht vor Ort, sondern nach einer Skizze angefertigt. Eine genauere Wiedergabe konnte nach der Neuausgrabung von La Rosa im Jahr 1997 gemacht werden: La Rosa 1999, Abb. 1.

25 R. Paribeni, Ricerche nel sepolcreto di Haghia Triada presso Phaestos, MonAnt 14, 1904, 717.

${ }^{26}$ La Rosa (Anm. 9:2000) 88 f.; ders. 1999, 179.

${ }^{27}$ La Rosa (Anm. 9:2000) 91.

${ }^{28}$ La Rosa 1999, 181 Abb. 5-6; ders., The painted Sarcophagus: determining the Chronology, in: Sherratt (Anm. 20) 996 f. 
Die Figurenfelder sind von bunten Streifen und Zonen mit Spiralen und Rosetten gerahmt, welche vermutlich Einlegearbeiten nachahmen. Auf der einst nach Norden orientierten Langseite (Seite A, Abb. 5) steht links eine Frau, offensichtlich eine Priesterin, die Blut oder Wein aus einem Gefäß in einen größeren Mischkrug zwischen zwei Doppeläxten gießt. Eine andere Frau trägt auf der Schulter eine Stange mit zwei weiteren Gefäßen, und ein Mann spielt die Lyra. Die zweite Szene stellt drei Männer dar, die Kultobjekte oder Gaben darbringen: zwei kleine Rinder und einen gekrümmten, nicht klar verständlichen $\mathrm{Ge}$ genstand, vermutlich ein Bootsmodell

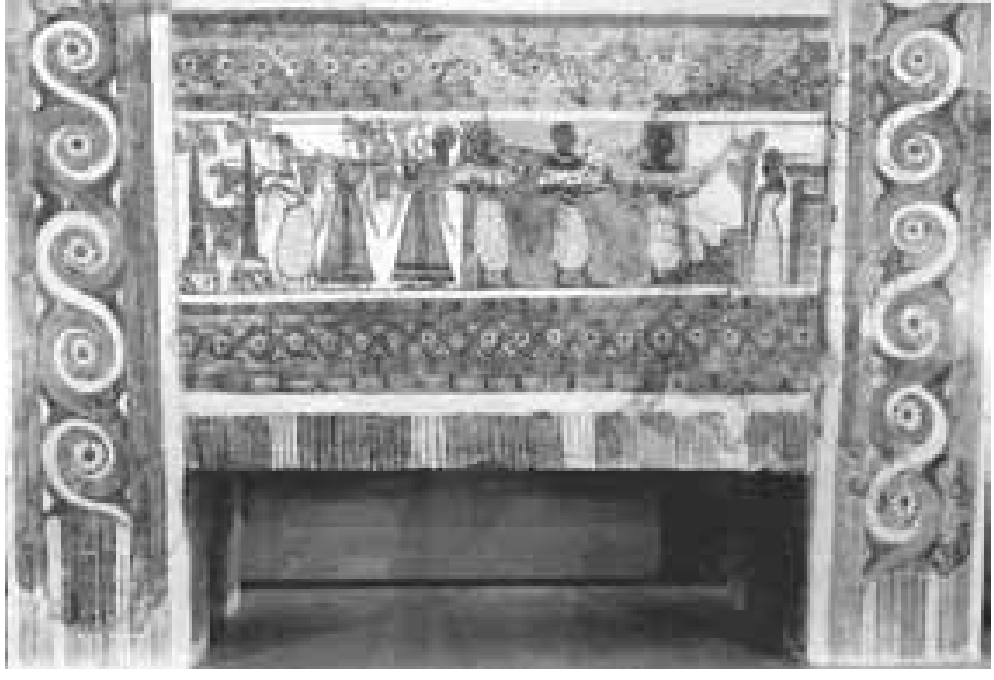

5 Sarkophag von Agia Triada, Seite A oder einen Elefantenstoßzahn. Diese Objekte werden von einem Mann entgegengenommen, der vor einer Gebäudefassade steht. Seine Arme sind nicht angegeben, und der untere Teil der Figur ist nicht erhalten. Zwischen den drei Trägern und der männlichen Figur befinden sich ein dreistufiger Bau und ein Baum. Im Zentrum der anderen Langseite (Seite B) ist ein Stier abgebildet, der auf einem hölzernen Tisch festgebunden ist. Zwei kleinere Tiere, wahrscheinlich Ziegen, warten indessen unter dem Tisch auf ihre Schlachtung. Ein Flötenspieler begleitet die Szene. Links schließt eine Gruppe von drei Personen an, während rechts eine Priesterin vor einem Altar steht. An den Schmalseiten ist jeweils ein Wagen dargestellt; einer wird von Greifen (Seite C), der andere (Seite D, Abb. 6) von Wildziegen oder Pferden ${ }^{29}$ gezogen. Im letzten Fall ist darüber noch eine weitere Szene fragmentarisch zu erkennen, die eine Prozession von mit kurzem Schurz bekleideten Männern zeigt; sie ist der großen knossischen Prozession sehr ähnlich.

\section{Der 'Freskenschutt’ ('Fossa degli Affreschi’)}

Den Darstellungen auf dem bemalten Sarkophag in vielerlei Hinsicht sehr ähnlich sind die zahlreichen Fragmente, die im Schutt unter einem großen mykenischen Gebäude, der sog. Casa VAP, gefunden wurden ${ }^{30}$. Der Schutt wurde in der Mitte der Periode SM IIIA2 aufgehäuft, als ein großflächiges Terrain für die große Stoa eingeebnet wurde. Die Freskofragmente stammen wahrscheinlich von einer SM-IIIA1-Ausstattung, d. h., sie sind älteren Datums als der Sarkophag und wurden in SM IIIA2 als Einfüllmaterial verwendet. Aufgrund ihres guten Erhaltungszustandes wurden sie wohl nicht als Folge einer Beschädigung von den Wänden entfernt, sondern im Zuge eines neuen Dekorationsprogramms abgenommen. Unglücklicherweise können wir nur zwei Szenen rekonstruieren: die 'Frau neben dem Altar' und die 'Große Prozession'.

Die Partie der 'Frau neben dem Altar' (Abb. 7) mit den Maßen $57 \times 42 \mathrm{~cm}$ lässt eine etwa 20-24 cm große Frauengestalt erkennen, die zwei Opfertiere, höchstwahrscheinlich Dammhirsche, zu einem Altar führt; die Szene ist von Rosetten eingerahmt. Dank ähnlicher Figuren auf Siegelringen aus Mykenä und Theben kann die Frauenfigur rekonstruiert werden ${ }^{31}$. Von der ‘Großen Prozession’ (Abb. 8), auf einer Fläche von 140

${ }^{29}$ J. P. Nauert, The Hagia Triada Sarcophagus. An iconographical Study, AntK 8, 1965, 91-98.

${ }^{30}$ Militello 1998, 81-85 (Funde); 132-148 und 185-224 (Fragmente); 283-308 (Ikonographie). Zur 'Casa VAP' s. Privitera (Anm. 10).

${ }^{31}$ Die alte, von Paribeni vorgeschlagene Rekonstruktion setzte den Altar rechts ins Bild, sodass sich die Frau und die Tiere von diesem Altar entfernten (Paribeni 1908, Abb. 22; Militello 1998, Abb. M). Später aber, vermutlich als nach dem Krieg die von D. Levi und N. Platon gestaltete Ausstellung im Archäologischen Museums von Herakleion eingerichtet wurde (s. das in den 50er Jahren gemalte Aquarell von Seuffert in: Militello 1998, Taf. 50 = hier Abb. 7), wurde eine neue und plausiblere Rekonstruktion vorgeschlagen: C. Long, The Ayia Triada Sarcophagus (1974) Abb. 35. Die von Younger vermutete Zugehörigkeit des Pigorini- 


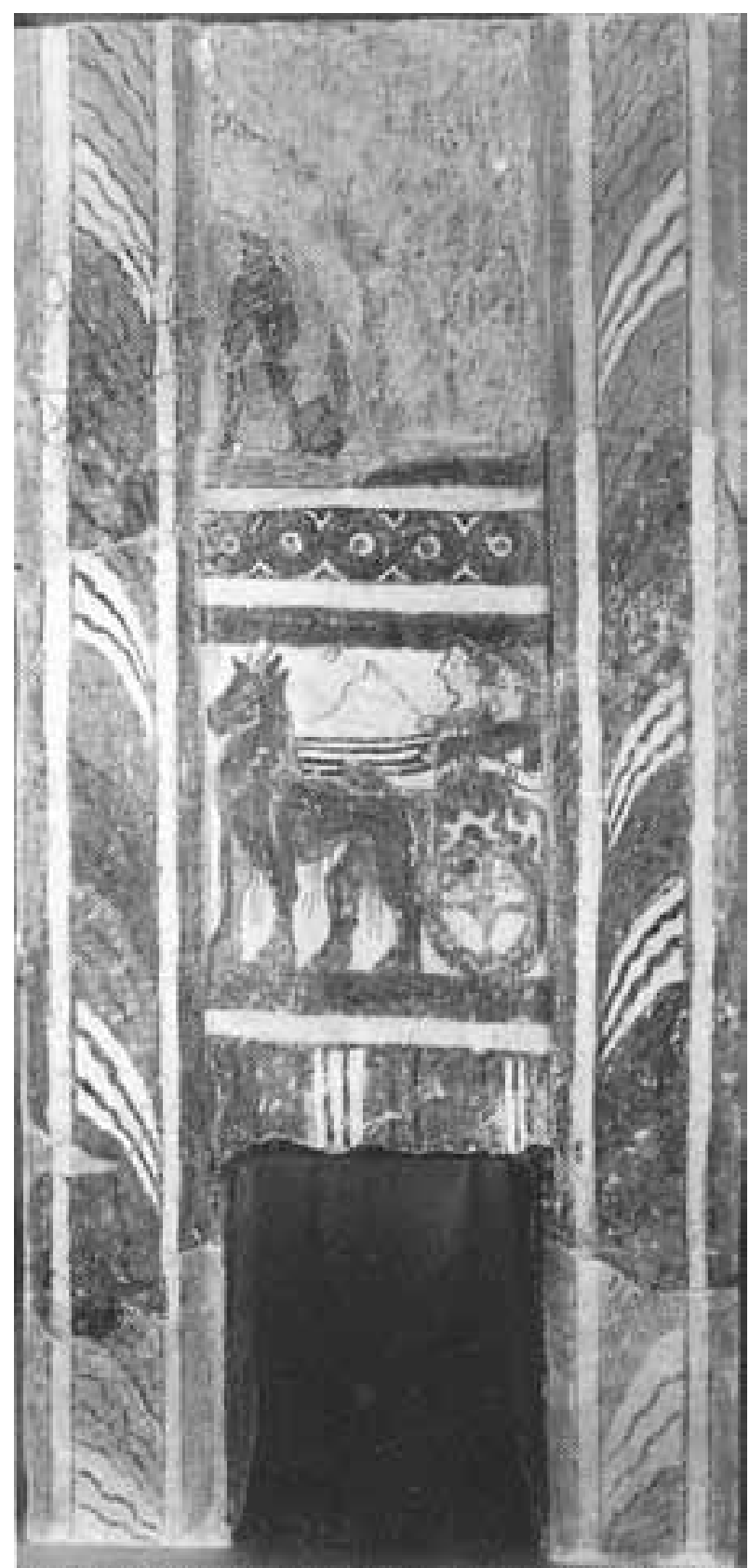

6 Sarkophag von Agia Triada, Seite D $\times 80 \mathrm{~cm}$ erhalten, lassen sich mindestens vier Figuren ergänzen, deren Größe zwischen 40 und $48 \mathrm{~cm}$ variiert. Hintergrund und Rahmen sind identisch mit dem zuvor behandelten Beispiel, obgleich die Figuren fast doppelt so groß sind (Abb. 12). Die Rekonstruktion im Archäologischen Museum von Herakleion zeigt eine Prozession von vier Figuren: einen Lyra- oder Phorminx-Spieler ${ }^{32}$, einen Gefäßträger, eine Gefäßträgerin sowie einen Flötenspieler. Nur der PhorminxSpieler und der Vasenträger sind eindeutig zu identifizieren, der Flötenspieler wurde hingegen anhand des Beispiels auf dem bemalten Sarkophag ergänzt. Unter den übrigen Fragmenten aus der 'Freskengrube' sind mehrere Themen zu erkennen, darunter Männer und Frauen in kostbarer Gewandung, manchmal in Gruppen dargestellt, die etwas darbringen (Abb. 9a). Auf einigen Fragmenten kann man auch Tiere, möglicherweise Wagen und laufende Athleten erkennen. Kultische Architektur bezeugt ein interessantes Fragment (Frgt. O3), auf dem ein Stufenaltar mit einem davor gelegenen Objekt dargestellt ist (Abb. 9b); das Objekt ist einem von A. Evans als »Omphalos « angesprochenen Motiv auf einem Wandmalereifragment aus Knossos sehr ähnlich ${ }^{33}$.

Aus all dem lässt sich schließen, dass es neben der 'Großen Prozession' und der 'Frau neben dem Altar' ursprünglich eine Reihe weiterer gerahmter, figürlicher Szenen gab, die an der Wand auf Augenhöhe, d. h. ca. 120-190 cm über Fußbodenniveau, angebracht war. Wie der am Rand des Frieses positionierte Lyraspieler der 'Großen Prozession' zeigt, wurde diese Darstellung in einem weiteren Bildfeld fortgeführt. Die Größe der Figuren schwankt und beträgt ca. 21, 28, 32 oder $48 \mathrm{~cm}$; die Frieshöhe blieb zweifellos konstant. Diese Beobachtungen implizieren, dass die Bilder Teil einer einheitlichen Dekoration waren, die zu einem einzigen Raum oder zwei eng miteinander verbundenen Räumen gehörte, wie z. B. im Fall des Hauptsaales und des Vorraumes eines Palastes. Die genauen Anbringungsorte der Fresken sind aber nicht mehr mit Sicherheit festzulegen. Die öffentlichen Bauten, die vor der 'Großen Stoa' errichtet worden waren und somit in Frage kämen, sind das Megaron, der Schrein und das 'Haus der enthaupteten Zimmer'; am wahrscheinlichsten ist sicherlich das große Megaron.

Die dargestellten Themen und die ikonographischen Motive auf den Fragmenten aus der 'Fossa degli Affreschi' sind mit denen des Sarkophags identisch. Technik und Stil sind jedoch qualitätsvoller und bestä-

Fragments zu diesem Bild ist nicht möglich, wie die stark abweichenden Dimensionen zeigen (s. hier Abb. 10): J. G. Younger, The Iconography of the Rulership in the Aegean: A Conspectus, in: P. Rehak (Hrsg.), The Role of the Ruler in the prehistoric Aegean. Proceedings New Orleans, Louisiana, 1992, Aegaeum 11 (1995) 164 Kat. 52 Abb. 56a (die Zeichnung beruht auf der Rekonstruktion von Stefani).

32 Das Musikinstrument ist von J. Younger, Music in the Bronze Age (1998), als Phorminx identifiziert worden.

33 PM II, 839. 555; D. Evely (Hrsg.), Fresco: A Passport into the Past. Minoan Crete through the Eyes of M. Cameron (1999) 205 Abb. 33c. 
tigen, dass hier nicht dieselben Künstler am Werk waren. Die meisten der Bilder stellen Kulthandlungen dar: Prozessionen von Frauen oder von Frauen und Männern, letztere im Allgemeinen mit spezifischen Aktivitäten wie dem Tragen von Objekten oder dem Spielen von Musikinstrumenten, der Opferung von Tieren, beispielsweise von Rindern, Stieren, Wildziegen und Cerviden, sowie der Darbringung von Opfergaben und Spenden auf einem Altar beschäftigt. Die Kulthandlungen spielen sich im Freien bei Altären und Einfriedungen ab. Einzelne ikonographische Motive, so das Tieropfer, der Wagen, die Agrimia und einige Gefäßtypen haben ihre Vorbilder in Beispielen der vorhergehenden Periode. Nur die 'Kleine Prozession' weicht von diesen Themen ab und scheint eine Zeremonie wiederzugeben, welche die sich von einander deutlich unterscheidenden Gruppen von Mädchen und Frauen einbezieht und wahrscheinlich im Inneren eines Gebäudes stattfand ${ }^{34}$. Diese Indizien bestätigen die Zugehörigkeit dieses Gemäldes zu einem anderen Dekorationsprogramm.

Die in den Fresken aus Agia Triada geschilderten Themen kehren auch auf Bildwerken in anderen Materialgattungen

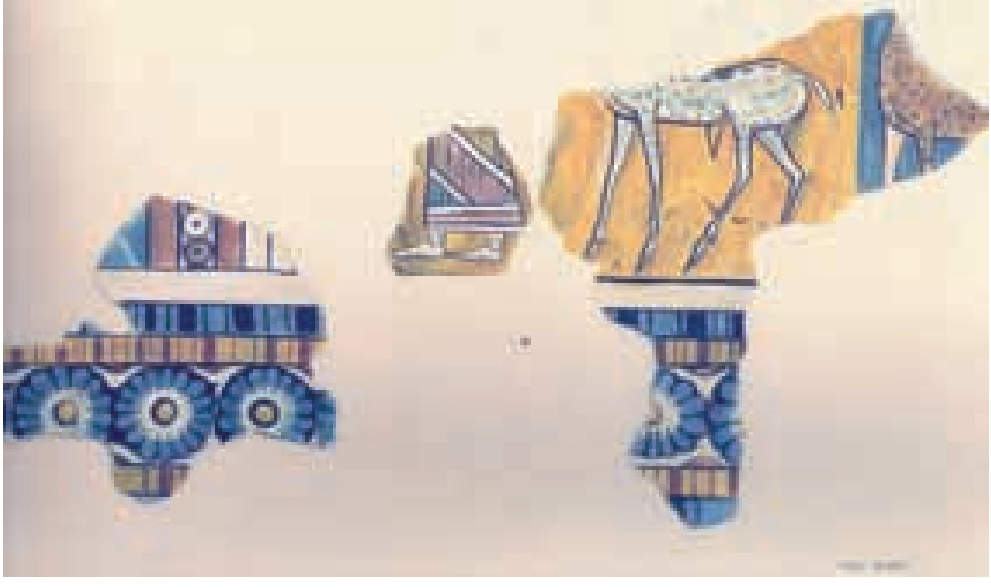

7 'Kleine Prozession’ aus Agia Triada

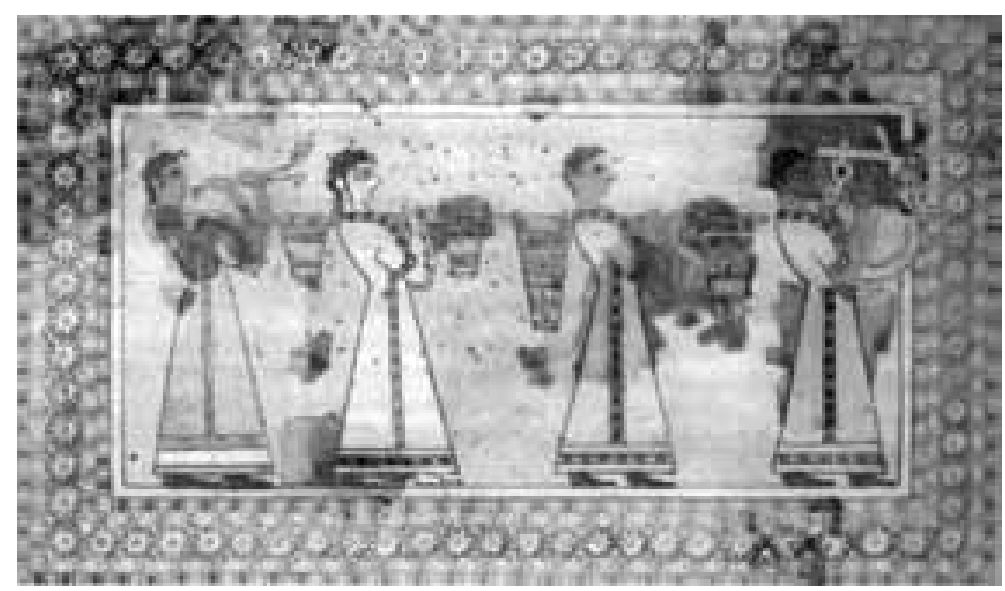

8 ‘Große Prozession’ aus Agia Triada und an anderen Orten Kretas wieder ${ }^{35}$. Bemerkenswert sind jedoch die ikonographischen Beziehungen zu Knossos, die am deutlichsten in der Prozession auf der einen Schmalseite des Sarkophags zu erkennen sind. Da die auf dem Sarkophag dargestellten rituellen Handlungen einem eher allgemeinen Repertoire angehören, das sich auch auf den sakralen Bereich erstreckte, kann deren ausschließlich sepulkrale Deutung, wie von R. Paribeni und M. Nilsson vorgeschlagen, nicht akzeptiert werden. Andererseits können wir vermuten, dass auf dem Sarkophag für den Verstorbenen

${ }^{34}$ Die 'Kleine Prozession’ zu interpretieren, ist nicht leicht. Es ist aber möglich, eine Art binäre Gegenüberstellung zwischen dem unteren und dem oberen Fries zu sehen, wenn auch durch ein sehr fragmentarisch erhaltenes Material. Im oberen tragen die Frauen langes Haar, eine Kopfbedeckung und eine üppige Brust; sie sind als isolierte Figuren auf gelbem Grund dargestellt. Im unteren Fries haben die Frauen rasierte Köpfe und sind als Gruppe auf violettem Grund gemalt. In der ägäischen Ikonographie sind rasierte Köpfe und nichtentwickelte Brüste typisch für junge Mädchen, während üppige Brüste und langes Haar Merkmale erwachsener Frauen darstellen. Folglich scheint der Unterschied der zwei Friese im Alter der Teilnehmerinnen zu liegen. Das würde eine entsprechende soziale Trennung von Erwachsenen und Minderjährigen widerspiegeln, die auch im Fall von Akrotiri auf Thera (Frauen in Xesté 3) offensichtlich ist.

${ }^{35}$ Vgl. z. B. zum Lyraspieler: Pylos, Tonplombe CMS I Nr. 36. Zum Greifenwagen: Siegel aus Anthia: CMS V Suppl. $1 B$ Nr. 137. Zum Agrimi-Wagen: Siegel aus Avdou in: V. E. G. Kenna, Cretan Seals, with a Catalogue of the Minoan Gems in the Ashmolean Museum (1960) Kat. 308 Taf. 12, 23 (= PM IV, 823 Abb. 803). Zum Tieropfer: Pylos, Hirsch und Altar: M. Lang, The Palace of Nestor at Pylos in Western Messenia. II. The Frescoes (1969) Kat. 3C20, Abb. 132. Das Stieropfer kehrt in mindestens 12 Siegelbildern wieder: s. den Katalog bei J. A. Sakellarakis, Das Kuppelgrab A von Archanes und das kretisch-mykenische Stieropferritual, PZ 45, 1970, 169-176 Abb. 8; Militello 1998, 295 f. Anm. 397; M. S. Speciale, La tavoletta PY Ta 716 e i sacrifici di animali, in: V. La Rosa - D. Palermo - L. Vagnetti (Hrsg.), Epi ponton plazomenoi. Simposio italiano di studi egei dedicato a L. Bernabò Brea e G. Pugliese Caratelli, Roma 1998 (1999) Abb. 1. 4-5. 

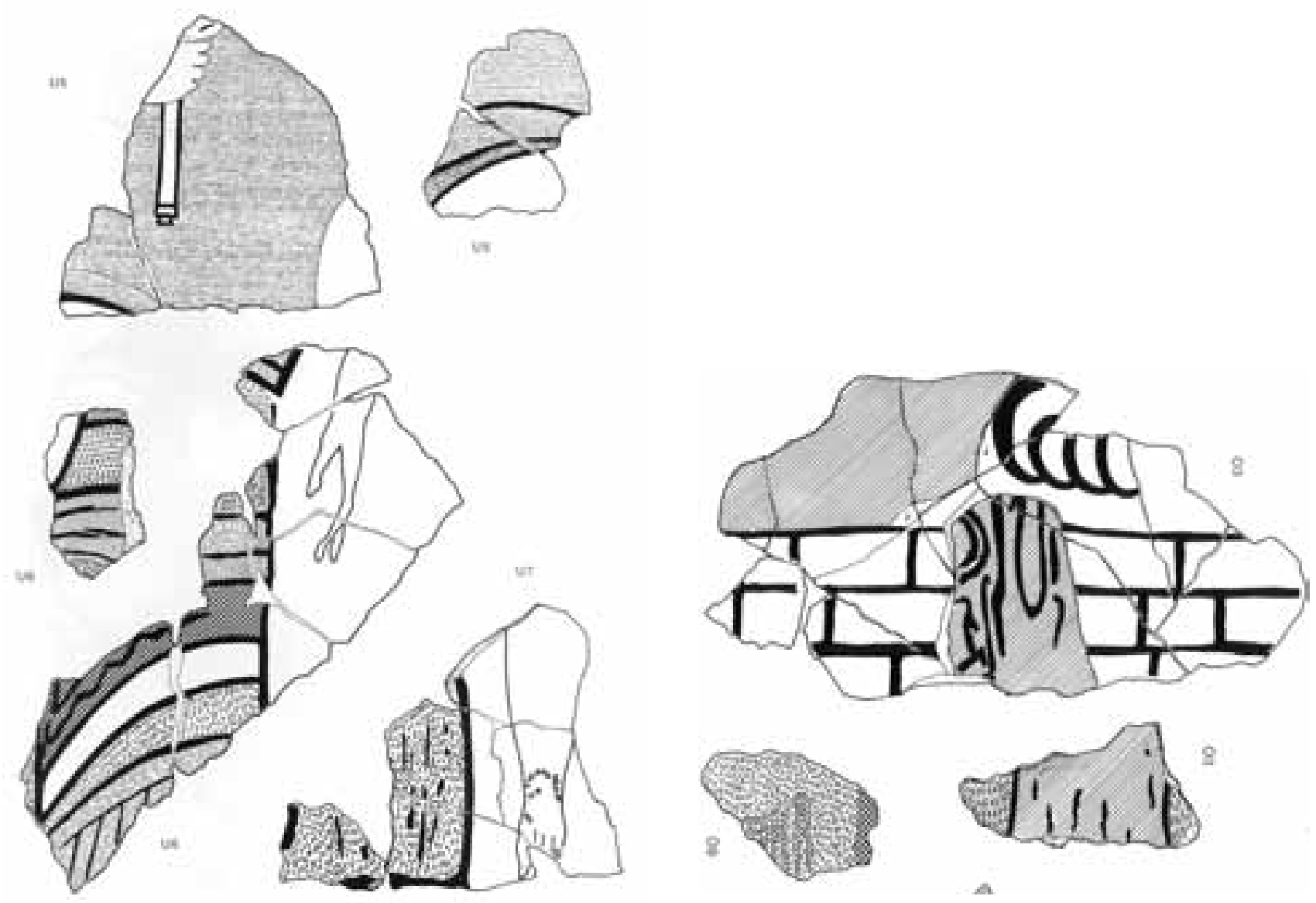

9a-b Fragmente aus der 'Freskengrube’ von Agia Triada
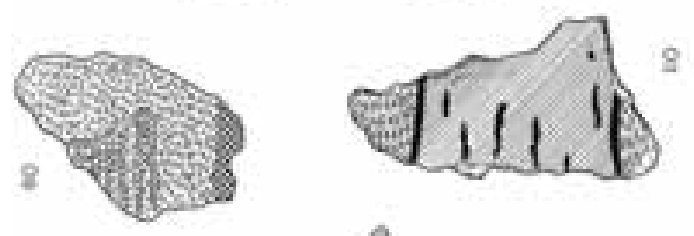

A

dieselben rituellen Handlungen dargestellt wurden, wie sie auch im Rahmen von Kultaktivitäten stattfanden. Es kann nicht ausgeschlossen werden, dass in Sonderfällen wie dem Tod eines bedeutenden, in diesem Grab beigesetzten Menschen ein aus dem sakralen Bereich entnommenes Ritual zelebriert und auf den Sarkophagwänden nach dem Vorbild der bereits existierenden Wandmalereien im Megaron abgebildet wurde.

\section{Kultaktivitäten}

Was die archäologischen Zeugnisse betrifft, bildete während SM IIIA der schon beschriebene Schrein den wichtigsten sakralen Bau in Agia Triada. Zur selben Zeit wurde auch ein weiterer kultischer Bereich benutzt, und zwar 'Vano E' (Abb. 1a) ${ }^{36}$. Hier wurden zwei 'Snake Tubes' gefunden, die in situ zurückgelassen worden waren, und sehr wahrscheinlich auch ein Tonidol sowie eine Kylix mit Oktopusdekor. Der Bau wurde in SM IIIA2 aufgegeben, möglicherweise zur selben Zeit, als mit der Errichtung der 'Großen Stoa' und des 'Hauses VAP' begonnen worden war. Und gerade im 'Haus VAP', im Raum A, wurden ein weiteres 'Snake Tube' und eine Pyxis deponiert, die S. Privitera als Zeugnisse eines Hauskultes interpretiert, obgleich eine offiziellere Funktion dieses Raumes nicht ausgeschlossen werden $\mathrm{kann}^{37}$. Es wäre möglich, dass einige Funde aus SM IIIB, die östlich der Stoa zutage kamen und die Existenz eines Kultortes nahelegen, in die letzte Phase des Schreins fallen. Hierbei handelt es sich um mindestens ein Idol vom Typus der 'Göttin mit erhobenen Armen'

\footnotetext{
${ }^{36}$ A. L. D’Agata, Un vano di culto TM III nell'abitato di Haghia Triada (Creta), Sileno 18, 1987, 135-145.

37 Banti (Anm. 14) 35 Abb. 21; S. Privitera, Culti domestici a Creta nel TM IIIA2-IIIB, Creta Antica 5, 2004, 166. Contra: Cucuzza 2003, 239.
} 
sowie um 'Snake Tubes' und Kalathoi, die vielleicht gemeinsam im Kult verwendet worden waren $^{38}$. Ab SH IIIC wurde das hypäthrale Heiligtum auf der 'Piazzale dei Sacelli' benutzt ${ }^{39}$; ein Zusammenhang mit den einst bestehenden Kultaktivitäten lässt sich nicht mehr feststellen.

Unter dem Fundmaterial im Bereich der Gräber im nordöstlichen Sektor begegnen uns einerseits die schon beschriebenen Grabbeigaben aus den Gräbern, andererseits aber auch Funde, die sich mit dem Kult in Verbindung bringen lassen: eine Plattform $\left(\mathrm{V}-\mathrm{V}^{\prime}\right)$ wurde in SM II für kultische (?) Zwecke benutzt; für SM III sind rituelle Aktivitäten im Bereich der sog. Tomba degli Ori anhand von Fragmenten einiger 'Champagne Cups' und eines amphoroiden Kraters (nördlich der Räume b, g) ${ }^{40}$ bzw. von Tassen, 'Champagne Cups' und Kylikes (in dem 'Piazzale lastricato' $)^{41}$ nachzuweisen. Obgleich die archäologischen Funde nicht sehr zahlreich sind, ist es doch sehr wahrscheinlich, dass sich der offizielle Kult hauptsächlich auf das Gebiet südlich der Ansiedlung konzentrierte, und nicht nur den Schrein, sondern auch das Megaron, den Kiosk, die Stoa und den davor gelegenen Platz einschloss. Aufgrund der späteren Wiederbenutzung kennen wir nur wenige Beifunde, die zur Ausstattung der SM-III-zeitlichen Gebäude gehörten. Eine religiö-
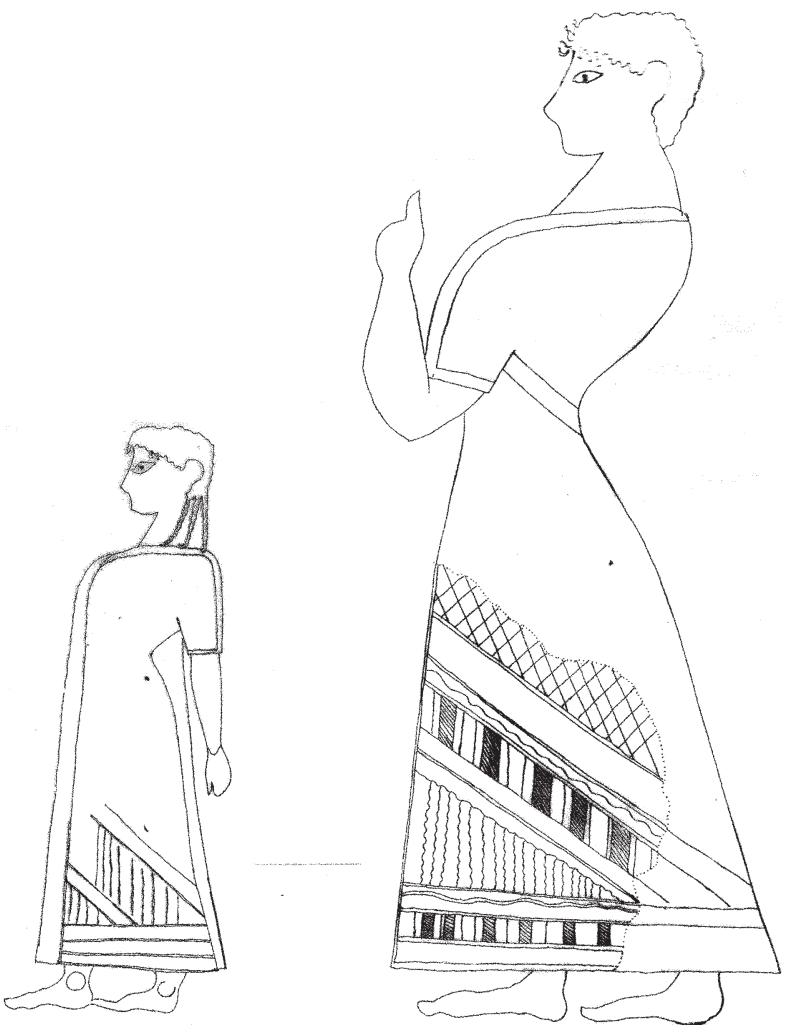

10 Figuren auf Fragmenten des Wandbildes der 'Frau neben einem Altar' und dem 'Fragment Pigorini' se Komponente ist auch für die rituellen Funktionen des Megarons in der mykenischen Welt gut vorstellbar und wird darüber hinaus in Agia Triada durch die Existenz von zwei sog. Steinmetzzeichen in Form einer Doppelaxt auf dem Pflaster vor dem Megaron ${ }^{42}$ sowie durch den Fund von Doppelaxtbasen (Abb. 11) zwischen dem Megaron und der Stoa wie auch nördlich davon unterstützt; eine dieser Basen trug ein aufgemaltes Greifenmotiv ${ }^{43}$. Außerdem wurden vermutlich einige Fragmente von Statuetten ursprünglich im Megaron selbst aufbewahrt ${ }^{44}$. Sowohl das Megaron als auch der Schrein richteten sich streng nach einem Planungskonzept: Letztgenannter Bau wurde exakt auf das Zentrum des Hauptgebäudes hin ausgerichtet und lässt vermuten, dass beide Bauten in enger Verbindung zueinander standen ${ }^{45}$.

\footnotetext{
${ }^{38}$ Zum Idol s. Banti (Anm. 14) 52 Abb. 30; D’Agata (Anm. 11:1999) 30 Kat. B7 (SM IIIB). Zu den gemeinsam mit Kat. B7 gefundenen 'Snake Tubes' s. Cucuzza 2003, 239 Anm. 170. Dieses muss in SM IIIB datieren. Aus dem östlichen Sektor des 'Muraglione a denti' kamen die Beispiele bei D’Agata (Anm. 11:1999) Kat. B3 (SM IIIA2) und B6 (SM IIIB), aus der 'Casa delle Camere Decapitate’: D’Agata (Anm. 11:1999) Kat. B12, 31-32.

39 D’Agata (Anm. 11:1999) 213-248; dies. (Anm. 11:2001).

40 Cucuzza 2003, 224 Abb. 8-9.

${ }^{41}$ Cucuzza 2003, 241. Zu den 'Snake Tubes': Banti (Anm. 14) 24. 35 (zwischen den Tholosgräbern).

${ }^{42}$ Dazu bereits Halbherr: N. Cucuzza, Mason’s Marks a Haghia Triada, Sileno 18, 1992, 53-65.

${ }^{43}$ Banti (Anm. 14) 16-18 Abb. 4; P. Militello, Archeologia, iconografia e culti ad Haghia Triada in età TM I, in: Laffineur - Hägg (Anm. 11) 160 Abb. 39a; Cucuzza 2003, 211 Abb. 6. Eine Tagebuchnotiz erinnert an den auf einer jetzt leider verschwundenen Basis gemalten Greifen: Militello 1998, 80. 359.

${ }^{44}$ D’Agata (Anm. 11:1999) 30 Kat. B13 (SM IIIA) und B9 (SM IIIB).

${ }^{45}$ N. Cucuzza, Religion and Architecture: early LM IIIA2 Buildings in the southern Area of Haghia Triada, in: Laffineur - Hägg (Anm. 11) 169-174 bes. $173 \mathrm{f}$.
} 


\section{Wandbilder und Kultaktivitäten in Agia Triada}

Wie zuvor dargelegt, stellen die Wandbilder aus Agia Triada Kultaktivitäten dar ${ }^{46}$. Die abgebildeten Rituale scheinen jedoch mit der archäologischen Evidenz nicht übereinzustimmen. Kylikes und Tassen, Tonidole und 'Snake Tubes', welche den Großteil der kultischen Fundgegenstände bilden, sind in den Fresken nicht dargestellt ${ }^{47}$. Andererseits sind die Paraphernalia und Kultvorrichtungen in den Wandbildern, wie treppenartige Altäre ${ }^{48}$, Opfertische, der Omphalos und die um einen Baum gebaute Einfriedung, in den archäologischen Zeugnissen aus Agia Triada nur schwer zu identifizieren ${ }^{49}$. Man kann lediglich mit einer gewissen Wahrscheinlichkeit davon ausgehen, dass das Grab und die angrenzende Baustruktur in Agia Triada mit der dekorierten Fassade und dem kleinen Altar auf Seite B des Sarkophags zu identifizieren sind. Die vergängliche Natur mancher Gegenstände könnte unter Umständen das Fehlen archäologischer Zeugnisse erklären. Es ist jedoch nicht zu übersehen, dass eine gewisse Diskrepanz zwischen den dargestellten und den tatsächlich gefundenen Gefäßtypen besteht. In seiner Analyse hat B. Burke den »altertümlichen« Charakter der auf dem Sarkophag gezeigten Gefäße betont ${ }^{50}$. So könnte man zu dem Schluss kommen, dass die auf den Wänden dargestellten Kultaktivitäten nur eine ikonographische Tradition und nicht das tatsächlichen Ritual widerspiegeln, wenn da nicht die Doppelaxtbasen wären, die auf dem Sarkophag dargestellt sind und jenen ähneln, die zwischen der Stoa und dem Megaron gefunden wurden. Dieser Fund bedeutet zweierlei: Einerseits zeigt

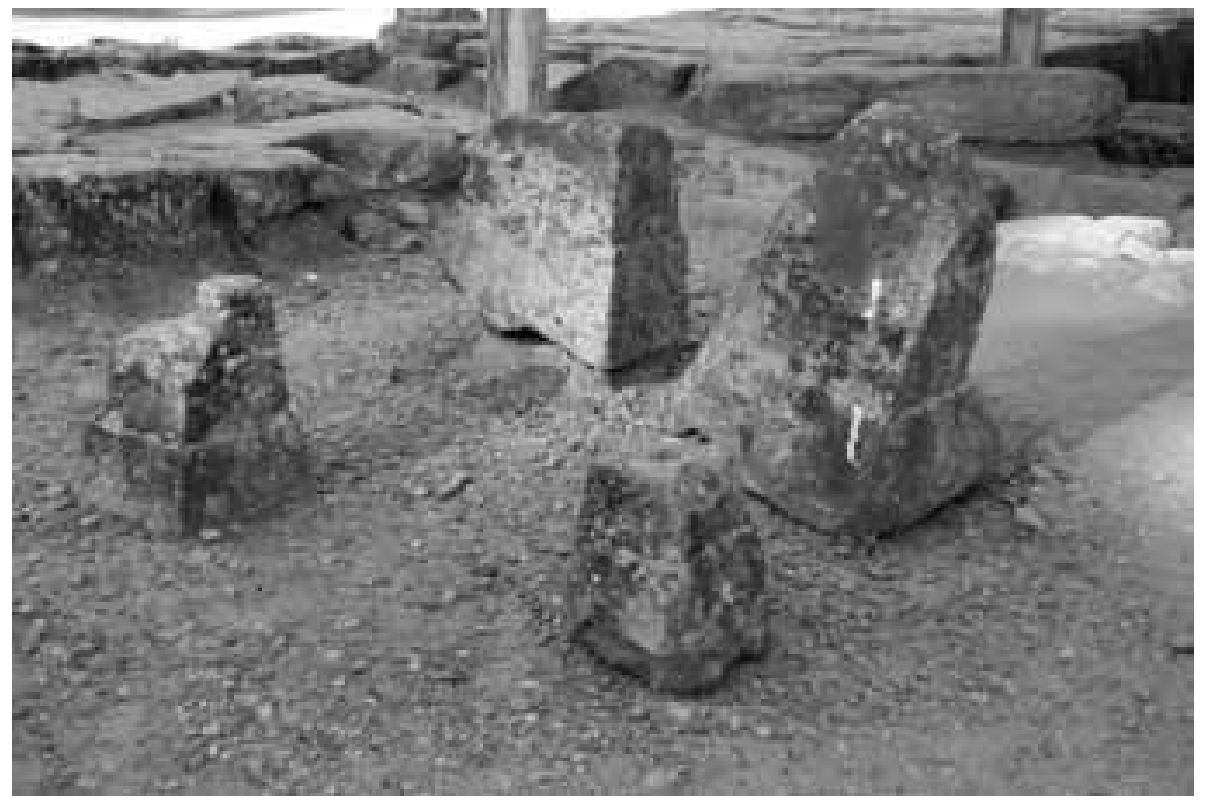

11 Doppelaxtbasen aus Agia Triada er eine gewisse Kontinuität der kultischen Handlungen des SM I in jüngerer Zeit, da Miniaturbasen aus der sog. königlichen Villa stammen. Andererseits bezeugt er, dass diese Aktivitäten in Agia Triada tatsächlich stattfanden und der wichtigste Ort des Geschehens gerade im südlichen Teil der Siedlung lag. N. Cucuzza hat sogar vermutet, dass es einen richtigen Prozessionsweg gab, der auch von Wagen befahrbar war, durch die 'Stoà ad L' führte und das Megaron mit dem Schrein verband $^{51}$. Wie oben beschrieben, setzt der innere

\footnotetext{
${ }^{46}$ Lediglich der bemalte Fußboden zeigt keine menschlichen Figuren, jedoch ist auch das Thema der Delphine und Oktopoden immerhin mit dem symbolischen und religiösen Wirkungsbereich verbunden.

${ }^{47}$ Nur ein Kalathos kann in der offenen Vase, die gegenüber der Priesterin auf der Seite B des Sarkophags dargestellt ist, erkannt werden.

48 Außerhalb von Haghia Triada ist ein treppenartiger Altar mit Sicherheit in dem in der Nachpalastzeit in der Nordwestecke des Zentralhofes in Phaistos zu identifizieren: L. Pernier - L. Banti, Il palazzo minoico di Festòs (1950) 55 f. Abb. 21-22; S. Alexiou,

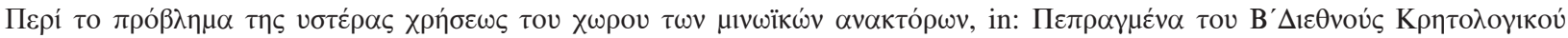

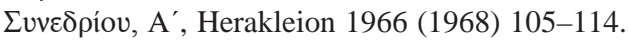

${ }^{49}$ Der Omphalos auf Fragment O2 kann vielleicht mit einem von netzartigem Flechtwerk oder Textilien überzogenen Stein identifiziert werden und in diesem Fall eine Entsprechung in den 'Baityloi' finden, die in der Nähe der Tholos A gefunden wurden: V. La Rosa, Minoan Baetyls: Between Funerary Rituals and Epiphanies, in: Laffineur - Hägg (Anm. 11) 221-227. Ihr Gebrauch ist aber nur für MM IA und MM II bezeugt.

50 Burke 2005, 413 f. Man muss aber betonen, dass der im 'Settore Nord-Est' gefundene Krater eine gewisse Ähnlichkeit mit dem von der Priesterin gehaltenen Krater, links der Seite A des Sarkophags, besitzt. Die Form, nicht jedoch die Dekoration des Kruges rechts auf der Seite B korrespondiert mit einem Fragment aus der 'Tomba degli Ori’: s. Paribeni (Anm. 25) 690 Abb. 6.

${ }^{51}$ Cucuzza (Anm. 45) 173 f.; Cucuzza 2003, 208. 215 f.
} 


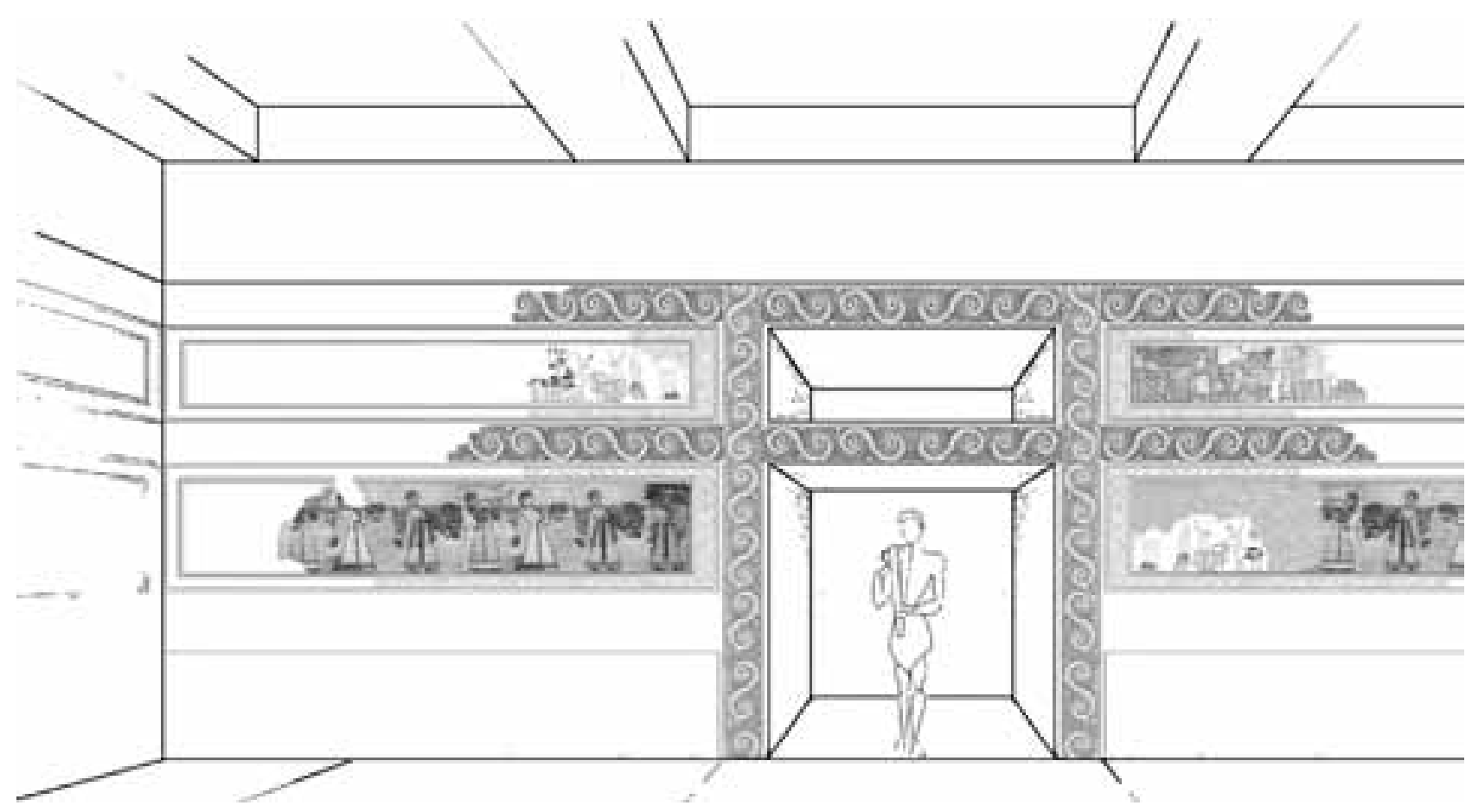

12 Rekonstruktionsvorschlag des Inneren des Megarons von Agia Triada

Raum des Schreins einen externen Betrachter voraus, wahrscheinlich die Teilnehmer der Prozession selbst. Nach dem Schrein und dem Megaron könnte das Gebiet der Nekropole - und hier speziell das bemalte Grab des Sarkophags - einen dritten Fokus auf dieser Prozessionsroute gebildet haben. Man darf vielleicht sogar vermuten, dass das Grab während besonderer Feierlichkeiten geöffnet und der bemalte Sarkophag zur Schau gestellt wurde.

Trifft diese Rekonstruktion zu, dann war jede Etappe der Prozession in der ersten Hälfte des 14. Jahrhunderts mit figürlichen Darstellungen geschmückt. Die Malereien im Schrein mit dem Thron unterstrichen die Verbindung zwischen der neuen politischen Macht und der Religion. Die Bilder an den Wänden des Megarons (Abb. 12) erinnerten an die rituellen Zeremonien, die auf der 'Piazzale dei Sacelli' vollzogen wurden. Die Verbindung zwischen diesen in verschiedenen Gebäuden angebrachten Malereien bekommt weitere Bestätigung in den Fresken des Megarons von Pylos, wo sich dieselben Themen wiederfinden. Hier wurden die Wände des Megarons mit Prozessionen, Rinderopferungen, rituellen Spenden und Lyraspielern bemalt, während auf dem Fußboden ein Oktopus dargestellt war ${ }^{52}$.

Es bleibt jedoch die Frage nach dem fehlenden Bezug der Malereien zum Gebrauch von Idolen und besonders zum Genuss von Nahrungsmitteln und Wein oder anderen Getränken, der in Agia Triada durch den Fund von Tassen und Kylikes bezeugt ist und bei den mykenischen Festen generell eine große Rolle spielte ${ }^{53}$. Möglich ist, dass sich die Funde aus dem Raum E oder der 'Casa VAP' auf eine unterschiedliche - private oder elitäre - Ebene der Religion beziehen, und es ist unbestritten, dass auch ein chronologischer Unterschied zwischen diesen Funden besteht. Es muss auch daran erinnert werden, dass Idole in Wandbildern aus Tiryns und Mykene begegnen ${ }^{54}$, Dreifüsse und Krüge in SM-I-Malereien in Pylos und Kylikes in den späteren Ge-

${ }^{52}$ C. W. Blegen - M. Rawson, The Palace of Nestor at Pylos in Western Messenia: I. The Buildings and their Contents (1966) Taf. 73 (Fußboden mit Oktopus aus dem Thronsaal); 173 (Fußboden mit Kraken und Delphinen aus Raum 50); Lang (Anm. 35) Taf. 119 (Vestibül); 125 (Thronsaal). Eine neue Rekonstruktion der Thronsaalfresken wurde von McCallum vorgeschlagen: L. McCallum, Decorative Program in the Mycenaean Palace of Pylos: The Megaron Frescoes (ph. D. University of Pennsylvania 1989).

53 J. Wright, A Survey of Evidence for Feasting in Mycenaean Society, in: ders. (Hrsg.), The Mycenaean Feast (2004) $133-178$.

${ }^{54}$ Tiryns: Ch. Boulotis, Zur Deutung des Freskofragmentes nr. 105 aus der Tirynther Frauenprozession, AKorrBl 9, $1979,59-67$.

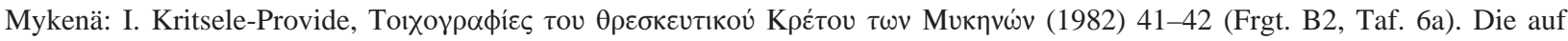
diesen Fragmenten dargestellten Idole ähneln aber nicht den bekannten Typen mykenischer Idole. 
mälden von Knossos wiederkehren ${ }^{55}$. Man kann auch davon ausgehen, dass die abgebildeten 'offiziellen' Rituale nach dem politischen Wandel in SM IIIA2 ein Ende gefunden hatten und durch andere kultische Handlungen ersetzt wurden. Es wäre jedoch ein Fehler, die Ikonographie nur als eine schlichte Widerspiegelung der Realität lesen zu wollen. Die Bilder sind ganz im Gegenteil eher eine Art Selbstdarstellung, die von der Wirklichkeit durchaus abweichen konnte, und somit diese Abweichung als Konsequenz einer Auswahl spezieller Themen erklärbar macht ${ }^{56}$. Auf jeden Fall ist bemerkenswert, dass die Sequenz der Bildszenen in Agia Triada auf das Tieropfer fokussiert und gerade dort endet, wo das Bankett beginnen müsste ${ }^{57}$. Dies könnte bloßer Zufall sein, jedoch ebenso gut auch auf einer Ablehnung der Darstellung des Speisevorgangs als Tabu oder aus Schamgefühl beruhen.

\section{Minoische Tradition und mykenische Innovation}

In der kurzen Zeit nach der Zerstörung der minoischen Paläste und nach der Eroberung Kretas durch die Mykener sind die Veränderungen, die den gesamten Bereich der materiellen und intellektuellen Kultur umfassen, offensichtlich. Im konkreten Fall von Agia Triada werden Elemente der Innovation vor allem in der Einführung neuer Architekturformen deutlich, wie uns das Megaron, die Stoa und der freistehende Schrein vor Augen führen. Im Bereich der Religion sind die Neuerungen in der regionalen Verbreitung der Heiligtümer, im Niedergang der sog. Bergheiligtümer, im unterschiedlichen Gebrauch der minoischen Paraphernalia und Symbole, besonders der 'Snake Tubes', und in der Einführung der sog. Göttin mit erhobenen Armen klar ersichtlich $^{58}$.

Für den Bereich der Malerei habe ich bereits vor einigen Jahren die verschiedenen ikonographischen, thematischen und stilistischen Veränderungen zusammengefass ${ }^{59}$. In der Ikonographie begegnen viele neue Elemente, die ein unterschiedliches kulturelles Milieu gegenüber jenem der Neupalastzeit zeigen: kurzes Haar von Männern, Raubvögel sowie das Zusammentreffen von Oktopoden und Delphinen. Andere Elemente wie die lange Männerkleidung, der Schurz ${ }^{60}$ und der dreistufige Altar existierten bereits in SM I, erfreuen sich aber jetzt einer größeren Beliebtheit. Bei dem in SM I-III häufigen Thema des Tieropfers ${ }^{61}$ ist die Bedeutung

\footnotetext{
55 Pylos: Lang (Anm. 35) Taf. 122. Knossos: 'Camp Stool Fresco’: S. Immerwahr, Aegean Painting in the Bronze Age (1990) Kat. Kn 26 (mit Bibliographie). Das Fragment ist bei M. Cameron - S. Hood, Arthur Evans’ Knossos Fresco Atlas (1974) Taf. 6, 1 abgebildet. Zur Datierung in SM IIIA2 oder SM IIIB s. S. Hood, Dating the Knossos Frescoes, in: L. Morgan (Hrsg.), Aegean Wall Painting. A Tribute to Mark Cameron (2005) 55 und Anm. 52. Eine Kylix wurde auch für die sitzenden Figuren in den Thronsaalfresken von Pylos vorgeschlagen: McCallum (Anm. 52).

${ }^{56}$ Eine ausführliche Analyse der in den 'Prozessionsfresken' dargestellten Gaben und ein Vergleich mit den Informationen der Linear-B-Texte sind neulich von F. Blakolmer durchgeführt worden (F. Blakolmer, Minoisch-mykenische 'Prozessionsfresken’: Überlegungen zu den dargestellten und den nicht dargestellten Gaben, in: E. Alram - G. Nightingale [Hrsg.], Keimelion. Elitenbildung und elitärer Konsum von der mykenischen Palastzeit bis zur homerischen Epoche. Internationaler Kongress aus Anlass des 65. Geburtstages von S. Deger-Jalkotzy, 3.-5. Februar 2005, in Salzburg, DenkschrWien 350 (2007) 41-57. Die Heterogenität und die Vielfältigkeit der Ikonographien führen den Autor zu dem Schluss, dass diese Fresken sich in zwei Gruppen teilen lassen: meist kleinformatige Opferprozessionen und großformatige Prozessionsfresken. Letztere sind mehr als Träger einer allgemeinen Symbolik von Prestige, die den palatialen Luxus wiederspiegelt, zu betrachten und nicht als Darstellungen richtiger Kulthandlungen.

${ }^{57}$ Bankett und Tieropfer sind zwei eng miteinander verbundene Aspekte der 'primitiven’ Mentalität: »Um die Verletzung der naturgegebenen Ordnung infolge des Tabubruchs der Tiertötung zu vermeiden, wird die Schlachtung rituell, d. h. als Opfer, vollzogen.« (J. Weilhartner, Mykenische Opfergaben nach Aussage der Linear B-Texte [2005] 13).

${ }^{58}$ Eine Übersicht über Kultstätten und Paraphernalia bietet G. C. Gesell, Town, Palace and House Cult in Minoan Crete, SIMA LXVII (1985) bes. 41: »... all the standard Minoan Cult Symbols ... continued to appear; however, the majority of these are not independent objects, but are attached to other cult objects. « Zu einer Analyse der Veränderungen in der Religion des SM III s. A. Peatfield, After the 'Big Bang « - What? Or Minoan Symbols and Shrines beyond Palatial Collapse, in: S. E. Alcock R. Osborne (Hrsg.), Placing the Gods. Sanctuaries and Sacred Spaces in ancient Greece (1994) 19-36. Zu den minoischen Höhenheiligtümern: E. Kyriakidis, Ritual in the Bronze Age Aegean. The Minoan Peak Sanctuaries (2005).

59 Militello 1998, 356-362.

60 s. aber P. Rehak, Aegean Breechcloths, Kilts, and the Keftiu Paintings, AJA 100, 1996, 35-51.

${ }^{61}$ Dazu grundlegend: J. A. Sakellarakis, Das Kuppelgrab A von Archanes und das kretisch-mykenische Stieropferritual, PZ 45, 1970, 135-198; s. auch die kritische ikonographische Analyse von R. Jung, Menschenopferdarstellungen? Zur Analyse minoischer und mykenischer Siegelbilder, PZ 72, 1997, 133-194 bes. 165-172.
} 
der Einführung des Dammhirsches als Ersatz für die Wildziege nicht zu unterschätzen. Der Hirsch ist in Anbetracht seines Wertes als königliches, mit Jagd, Landbesitz und Tapferkeit assoziiertes Symbol ${ }^{62}$ und seiner Rolle im Opferritual ${ }^{63}$ vermutlich einem festländischen Einfluss zuzuschreiben. Die größte Veränderung in der Freskenkunst kann man aber in der Wahl der Bildthemen erkennen: die Landschaften, die Epiphanien der Gottheit und die großen kommunalen Feste sind nun verschwunden. Das vorherrschende Thema bilden jetzt die Prozession und die damit verbundenen Aktivitäten. Diese Neuerungen spiegeln sich auch im Stil wider: Die Erstarrung der Figuren, der Mangel an Landschaftsangaben und der monochrome Hintergrund, verbunden mit der Gliederung der Szenen in verschiedenfarbigen Feldern - gelb, blau, weiß; rot nur in der Greifenwagenszene $^{64}$-, können ihre Rechtfertigung in dem Anspruch auf eine klarere Darstellungsweise finden.

Im Allgemeinen wurden diese neuen Elemente in Architektur, Kunst und Brauchtum auf Kreta in der Forschung als Folge des Eindringens der Mykener erklärt, weshalb man versucht hat, eine Gegenüberstellung zwischen 'minoisch' und 'mykenisch' in der materiellen Kultur vorzunehmen ${ }^{65}$. Im speziellen Fall von Agia Triada und der Messara hat A. L. D’Agata vor wenigen Jahren die keramischen Komplexe analysiert und festgestellt, dass, während die Keramik aus der Siedlung und aus den »Gräbern der Reichen« in Kalyvia viele stilistische Gemeinsamkeiten mit jener der mykenischen »Herrenschicht« in Knossos zeigt und »knossische« Bewohner voraussetzt, die Funde aus der Umgebung eher in der minoischen Tradition verhaftet geblieben zu sein scheinen ${ }^{66}$. 2003 verglich N. Cucuzza die Funde der verschiedenen Kultorte in der Nähe von Agia Triada miteinander und erkannte einen Gegensatz zwischen den Fundkomplexen in der minoischen und jenen in mykenischer Tradition. Die konischen Becher aus dem Schrein und die Larnakes der Nekropolen gehören der minoischen Tradition an, während sich die Kylikes aus Raum E und aus der Nekropole von Kalyvia sowie die Holzbahren (ebenfalls aus Kalyvia) mit der mykenischen Tradition verbinden lassen ${ }^{67}$. Ein Jahr später erklärte L. Preston in ihrer Analyse der Grabsitten der SM-II-III-Oberschicht die Funde aus dem SM-IIIA-zeitlichen Agia Triada - und besonders das Grab des Sarkophags - als das Ergebnis einer interregionalen Konkurrenz zwischen den Eliten ${ }^{68}$ : »This unique artifact (i. e. the Sarcophagus) encapsulates the desire for display and the willingness to experiment and innovate that characterize these elite cemeteries in this horizon. « Schließlich hat B. Burke bei seiner Studie zum Sarkophag von Agia Triada herausgefunden, dass mehrere hier dargestellte Vasen aus der MM- und SM I-Tradition stammen und diese »intentional fusion of mainland and Cretan elements « mit dem Bedürfnis eines mykenischen Herrschers, seine Macht bei der minoischen Bevölkerung zu legitimieren, erklärt werden kann ${ }^{69}$.

\footnotetext{
${ }^{62}$ C. E. Morris, In Pursuit of the white tusked Boar: Aspects of Hunting in Mycenaean Society, in: R. Hägg - G. C. Nordquist (Hrsg.), Celebrations of Death and Divinity in the Bronze Age Argolid. Proceedings of the 6th International Symposion at the Swedish Institute at Athens 1988 (1990) 149-156; M. Cultraro, Exercise of Dominance. Boar Hunting in Mycenaean Religion and Hittite Royal Rituals, in: M. Hutter - S. Hutter-Braunsar (Hrsg.), Offizielle Religion, lokale Kulte und individuelle Religiosität. Akten Bonn 2003 (2004) 117-135.

${ }^{63}$ F. Brein, Der Hirsch in der griechischen Frühzeit (Diss. Wien 1969) (Täfelchen PY Cn 591, 868+875; Ideogramme *104). Zum Tieropfer in den mykenischen Texten s. auch Weilhartner (Anm. 57) 156. Hirschknochen sind die am häufigsten gefundenen

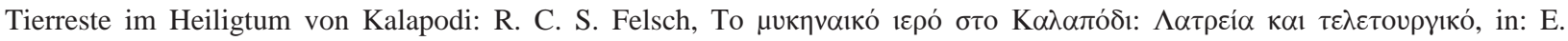
Froussou (Hrsg.), The Periphery of the Mycenaean World. 1st International Congress Lamia 1994 (1999) 163-170. In Agia Triada gibt es in SM IIIA eine größere Menge davon als in SM I oder im MM; dazu B. Wilkens, I resti faunistici di Haghia Triada (Creta) in età neo e postpalaziale: Nota preliminare, in: De Miro - Godart - Sacconi (Anm. 23) III, 1511-1520; dies., The Fauna from Italian Excavations on Crete, in: D. S. Reese (Hrsg.), Pleistocene and Holocene Fauna of Crete and Its First Settlers, Monographs in World Archaeology 28 (1996) 241-261.

${ }^{64}$ Vielleicht ist diese Farbwahl bloß einer Vorliebe für Variationen zuzuschreiben. Nach Ansicht von Pötscher (Anm. 23:1997) besitzen die Farben hier eine semantische Funktion, z. B. als zeitliche Kennzeichnung.

65 s. die Diskussion bei W.-D. Niemeier, Die Palaststilkeramik (1985) 217-233. Zur Religion s. außer den o. in Anm. 58 zitierten Werken C. Renfrew, Questions of Minoan and Mycenaean Cult, in: R. Hägg - N. Marinatos (Hrsg.), Sanctuaries and Cults of the Aegean Bronze Age (1981) 27-33; ders., The Archaeology of Cult. The Sanctuary at Phylakopi (1985) 435-444.

66 A. L. D’Agata, Dinamiche sociali, modelli culturali e indicatori etnici a H. Triada nel TM III. L'evidenza offerta dalla ceramica, in: La Rosa - Palermo - Vagnetti (Anm. *) 189-198; dies., Hidden Wars: Minoans and Mycenaeans at Haghia Triada in the LM III Period. The Evidence from Pottery, in: R. Laffineur (Hrsg.), Polemos. Actes de la 7e Rencontre égéenne internationale, Université de Liège, 14-17 avril 1998(1999) 47-55.

${ }^{67}$ Cucuzza 2003, $237 \mathrm{f}$.

${ }^{68}$ L. Preston, A mortuary Perspective on political Changes in LM II-IIIB Crete, AJA 108, 2004, 321-348 (Zitat auf S. 336).

${ }^{69}$ Burke 2005, 418: »... an ideological tool of a newly installed Mycenaean elite.«
} 


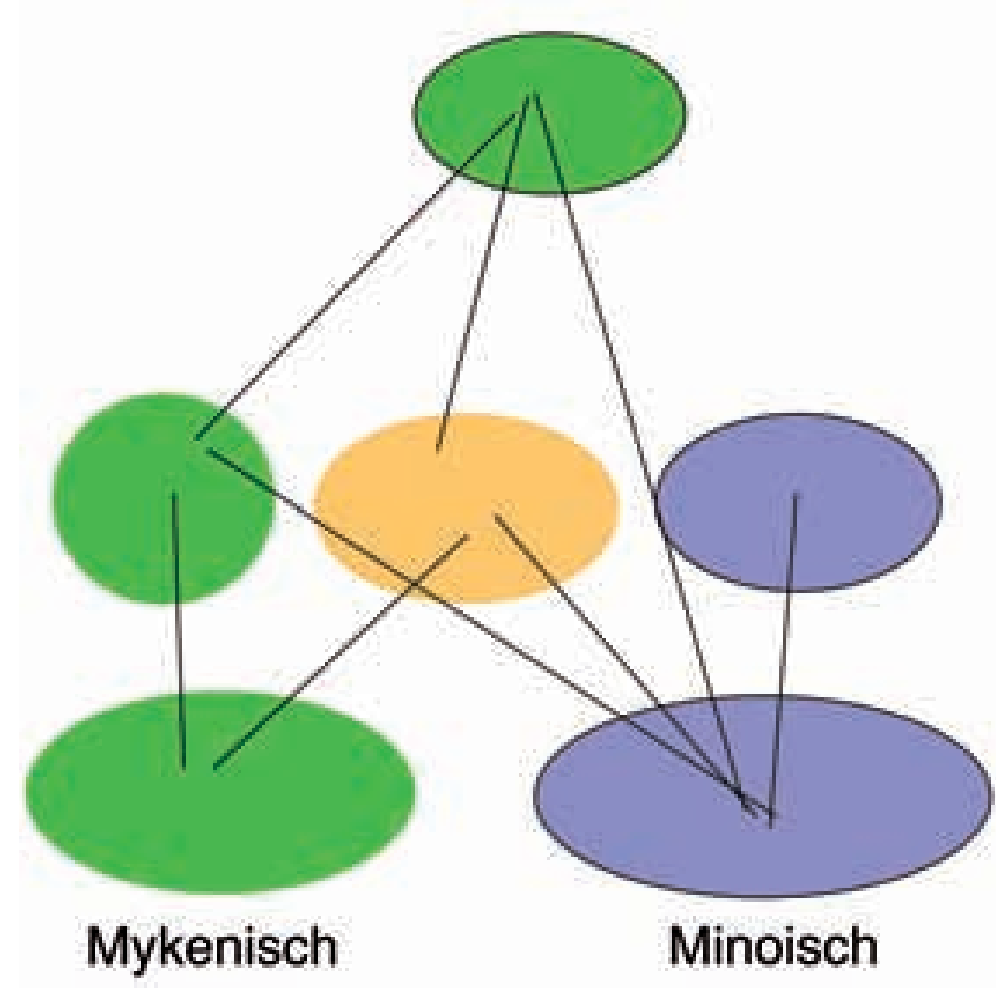

Diagramm 1: Gesellschaftliche Stratifikation Kretas in SM II-IIIA1
Diese Ergebnisse sind jedoch nicht immer so eindeutig, und, wie L. Preston zu Recht betonte, könnten diese Neuerungen eher als Ergebnis eines Experimentierens und Konkurrierens denn als bloßer Ausdruck ethnischen Charakters verstanden werden. Wie aus der Untersuchung von N. Cucuzza deutlich hervorgeht, ist nicht zu leugnen, dass manche Funde eine klare und absolute Gegenüberstellung nicht erlauben: So wurde z. B. eine mykenische Kylix auch in der minoischen Tholos von Kamilari gefunden, die minoischen konischen Becher des Schreins wurden in einem architektonischen Kontext entdeckt, der eine Neuerung darstellte, und die Meeresmotive tauchen auf dem Fußboden des Schreins und auf der Kylix aus Raum E auf. Die 'Snake Tubes' wiederum sind in allen religiösen Kontexten zu finden. Außerdem wurde das Megaron an demselben Ort wiederaufgebaut, an dem bereits zuvor die minoische Villa stand, und auch dieselbe gepflasterte SM-I-Esplanade wurde später wiederbenutzt. Diese Beispiele einer substanziellen Ambiguität in der Interpretation zeugen von der Schwierigkeit, ethnische Unter-
schiede lediglich aufgrund archäologischer Zeugnisse zu fassen. Dies gilt besonders für die Hofkultur, die offizielle Ikonographie sowie die öffentlichen Kulthandlungen, und zwar aus zwei Gründen:

Erstens war dieselbe 'mykenische Kultur' schon in SH II auf dem griechischen Festland stark 'minoisiert', zumindest auf der Ebene der Eliten. Themen und Ikonographie der minoischen Tradition wurden assimiliert, weshalb es schwer ist festzustellen, wieviel sich in SM III noch direkt von der minoischen Tradition ableitet und wie viel von Mykenern vermittelt wurde. Man muss zudem betonen, dass die Bildsprache des SM III keine gänzliche Neuerung darstellt, sondern das Resultat eines Prozesses ist, der bereits ein Jahrhundert zuvor einsetzte und mit dem Wandel von einem minoischen zu einem verstärkt bürokratischen Staat wie dem mykenischen zusammenhängt. Auf diese Weise können wir auch die Übernahme ägyptischer Themen und Motive erklären, die in der Malerei dieser Zeit sehr häufig begegnen, wie St. Hiller darlegte ${ }^{70}$. Diese gehen auf die Notwendigkeit der ägäischen Eliten zurück, eine Bildsprache zu schaffen, die dieser neuen Form des Staates besser entsprach.

Das zweite Hindernis, das eine eindeutige Auslegung erschwert, stellen die Individuation zweier entgegengesetzter ethnischer Einheiten dar sowie die Möglichkeit, diese aus der materiellen Kultur heraus zu bestimmen. Nimmt man die jüngsten Studien zur ethnischen Identität zum Ausgangspunkt ${ }^{71}$, so erscheint es angebracht, die 'ethnischen Realitäten' nicht als starre, feste Blöcke zu betrachten. Dies gilt generell für Prozesse der Interaktion zwischen Eliten oder zwischen Zentrum und Peripherie, wie etwa Studien über die Romanisierung der Provinzen gezeigt haben ${ }^{72}$. In der Peripherie kann es Gruppen geben, welche die Absicht haben, sich an das Zentrum anzupassen, und im Gegensatz dazu Eliten, die dazu neigen, ihre Unabhängigkeit stärker zu behaupten. So können wir die Gesellschaft Kretas (Diagramm 1) am Anfang des 14. Jahrhunderts

${ }^{70}$ St. Hiller, Zur Rezeption ägyptischer Motive in der minoischen Freskenkunst, AegLev 6, 1996, 85-106; ders., Egyptian Elements on the Hagia Triada Sarcophagus, in: P. Betancourt u. a. (Anm. 23) 361-368.

71 S. Jones, The Archaeology of Ethnicity (1997); J. M. Hall, Ethnic Identity in Greek Antiquity (1997); J. M. Hall u. a., Ethnic Identity in Greek Antiquity, Cambridge Archaeological Journal 8, 1998, 265-283.

${ }^{72}$ M. Millett, The Romanization of Britain (1990); J. Webster, Creolizing the Roman Provinces, AJA 105, 2002, $209-225$. 
als eine Pyramide betrachten, die auf dem unteren Niveau, jenem der sozialen Unterschicht, zwei abgegrenzte Gruppen enthielt: die minoische und die mykenische, welche anhand der materiellen Kultur greifbar werden.

An der Spitze befand sich eine herrschende mykenische Schicht. Die mittlere Stufe aber ist sehr verschiedenartig strukturiert: Es gab mykenische Beamte, den minoischen Adel, der sich an die zentrale Macht anpassen wollte, weiters minoische Eliten, die ihre Identität bewahren wollten und sogar ihre Oppositionshaltung zu bekräftigen versuchten und deren kulturelle Lebendigkeit etwa durch die SM-III-zeitliche Linear A-Inschrift auf einer Statuette aus Poros bezeugt wird ${ }^{73}$.

Besser, als das Problem nun in ein enges ethnisches Modell zu pressen, wäre es, in der Interpretation der offiziellen Kunst und Religion von einer Dialektik der konkurrierenden Gruppen zu sprechen, die manchmal ihre Tradition beanspruchten oder sich auf die zentrale Macht beriefen, d. h. zwischen Zentrum und Peripherie, Tradition und Innovation wechselten ${ }^{74}$. Alle Formen symbolischer Repräsentation der politischen Ideologie in den zwei Phasen des SM IIIA in Agia Triada, von der Architektur bis zur Malerei, drücken zweifelsohne eine Anpassung an die neue Zentralmacht aus, indem deren Bildsprache imitiert wird. Gleichzeitig erkennen wir den Versuch, die alte Tradition der minoischen Villa wiederaufzunehmen. Ob dies von einem mykenischen Herrscher initiiert wurde, der mit der Vergangenheit Kretas seine Macht rechtfertigen wollte, oder von einem minoischen, der das Wohlwollen von Knossos auf sich ziehen wollte, können wir nicht sagen. Die komplette Veränderung des Stadtplans in der dritten Phase, die Entfernung der Wandbilder, die Schändung des Sarkophags und vielleicht sogar die Verbrennung des Schreins weisen hingegen auf die Präsenz einer neuen herrschenden Gruppe hin, die sich nicht nur durch die Zerstörung der Machtsymbole der vorhergehenden Eliten, sondern auch durch die Vernichtung jeglichen Bezugs auf die knossische Macht unterscheiden wollte.

Prof. Dr. Pietro Militello

Centro Archeologia Cretese, Dip. SAFIST, Università di Catania, Via A. di Sangiuliano 262, I-95124 Catania

E-Mail:milipi@unict.it

Abbildungsnachweis: Abb. 1a-c: Nach Archiv CAC; Abb. 2: nach Militello 1998, Abb. 38; Abb. 3: nach Militello 1998 , Taf. 10, a; Abb. 4: nach La Rosa 1999, Abb. 9; Abb. 5: nach Militello 1998, Taf. 14 a; Abb. 6: nach Militello 1998, Taf. 16, a; Abb. 7: nach Militello 1998, Taf. 50; Abb. 8: Photo M. Quaresima, 1990; Abb. 9a-b: nach Militello 1998, Abb. 24. 26; Abb. 10: Rekonstruktion Verf.; Abb. 11: Photo Militello, 2005; Abb. 12: Rekonstruktion Verf.

\footnotetext{
${ }^{73}$ N. Dimopoulou - J.-P. Olivier - G. Réthémiotakis, Une statuette en argile avec inscription en linéaire A de Poros/Irakliou, BCH 117, 1993, 501-521.

${ }^{74}$ s. auch meine Überlegungen dazu: Militello 1998, 352-354; ders., O1 $\mu$ (Anm. 63) 349-350.
} 
